\title{
Orbital stability for polytropic galaxies
}

\section{Stabilité orbitale pour les galaxies polytropiques}

\author{
Óscar Sánchez, Juan Soler* \\ Departamento de Matemática Aplicada, Facultad de ciencias, Universidad de Granada, 18071 Granada, Spain
}

Received 11 March 2004; received in revised form 5 May 2005; accepted 17 February 2006

Available online 22 September 2006

\begin{abstract}
In this paper, motivated by the Galilean invariance of the solutions, we use the concept of orbital stability for the Vlasov-Poisson system. We prove that a family of stationary solutions, called polytropic gas spheres, are orbitally $L^{1}$ stable in the gravitational case by considering direct variational arguments.

(C) 2006 L'Association Publications de l'Institut Henri Poincaré. Published by Elsevier B.V. All rights reserved

\section{Résumé}

Dans cet article, motivés pour la propriété d'invariance Galiléenne des solutions, nous étudions la notion de stabilité orbitale pour le système de Vlasov-Poisson. Nous obtenons pour le cas gravitationnel que la famille des solutions stationnaires, connue comme gaz de sphères polytropiques, sont orbitalement stables dans $L^{1}$ en considérant des arguments variationnels directs.

(C) 2006 L'Association Publications de l'Institut Henri Poincaré. Published by Elsevier B.V. All rights reserved

MSC: primary 35A15, 35B40, 82B40, 82C40; secondary 35B35, 35B45, 35J20, 35J60, 76P05, 82D99

Keywords: Vlasov-Poisson system; Stellar dynamics; Polytropic gas spheres; Gravitation; Orbital stability; Galilean invariance; Concentration compactness; Direct variational methods
\end{abstract}

\section{Introduction and main result}

This paper relies on the analysis of stability properties of stationary solutions, called polytropic spheres, to the VP system in the gravitational case

$$
\begin{aligned}
& \partial_{t} f+v \nabla_{x} f-\nabla_{x} \phi \nabla_{v} f=0, \\
& f(t=0, x, v)=f^{0}(x, v),
\end{aligned}
$$

\footnotetext{
This research was partially supported by the EU financed network IHP-HPRN-CT-2002-00282, by MCYT (Spain) proyectos BFM2002-00831, MTM2005-02446 and Junta de Andalucía proyecto de excelencia FQM792.

* Corresponding author.

E-mail addresses: ossanche@ugr.es (Ó. Sánchez), jsoler@ugr.es (J. Soler).
} 


$$
\Delta_{x} \phi=4 \pi \rho, \quad \lim _{|x| \rightarrow \infty} \phi(t, x)=0 .
$$

This topic is of particular interest in stellar dynamics for the understanding of galaxies and clusters.

The existence of families of stationary solutions to the gravitational VP system has been previously analyzed in several works, e.g. $[5,16]$. Here, we focus our attention on a particular family, the so called polytropic spherical systems [16], which are defined by

$$
v_{\mu}=v(x, v)=c\left(E_{0}-|v|^{2} / 2-\phi(|x|)\right)_{+}^{\mu},
$$

where $(f)_{+}$denotes the positive part of $f, \alpha<E_{0}<0,-1<\mu$ and $c>0 . \phi$ is coupled with $v$ by the Poisson equation (1.3) and satisfies $\lim _{|x| \rightarrow 0} \phi(|x|)=E_{0}-\alpha$. The family (1.4) involves four different parameters. However, the Coulombian constraint implies the existence of a unique polytrope for any admissible values of $\mu, c$ and $\alpha$. Typically, these solutions are indexed by the exponent $\mu$. The functions represented by (1.4) can be seen as a particular case of the family of generalized polytropic solutions $[5,16]$. The existence of these and other families of solutions were proved in [5] by means of the associated characteristic system.

A wide literature could be mentioned from the 60's about the stability properties of these solutions. In [1,2] Antonov studied the linear stability of polytropic solutions with $0<\mu<7 / 2$. Later, in $[3,16]$ the stability for other values of $\mu$ was analyzed from a numerical point of view. Several works develop nonlinear dynamical stability criteria $[8,11-14,19,20,23]$ for some of these solutions via variational arguments based on the Energy-Casimir or the Energy functionals. In this direction, in $[11,13,20,23]$ the polytropes defined by $0<\mu<\frac{3}{2}, c=1$ and $\int_{\mathbb{R}^{3}} v_{\mu} \mathrm{d} x=M>0$ were deduced as the minimizers of the Energy-Casimir functionals

$$
Q_{\mu, k}(f)=\frac{\mu}{\mu+1} \int_{\mathbb{R}^{6}} f^{1+1 / \mu} L^{-k / \mu} \mathrm{d} x \mathrm{~d} v+E(f),
$$

in a mass constrained space, i.e. $\|f\|_{\mathrm{L}^{1}\left(\mathbb{R}^{6}\right)}=M>0$, where $L=|x \times v|^{2}, k=0$ and the Total Energy functional $E(f)$ is defined by

$$
E(f)=\frac{1}{2} \int_{\mathbb{R}^{6}}|v|^{2} f(t, x, v) \mathrm{d} x \mathrm{~d} v-\frac{1}{8 \pi} \int_{\mathbb{R}^{3}}|\nabla \phi|^{2} \mathrm{~d} x .
$$

Here, the first term in the right-hand side is the kinetic energy $E_{\mathrm{KIN}}(f)$ and (minus) the second term is the potential energy $E_{\mathrm{POT}}(f)$. This result was also proved for generalized polytropic solutions under the additional assumption that $f$ is also spherically symmetric. The total energy, as well as the norms $\|f\|_{L^{p}\left(\mathbb{R}^{6}\right)}$ with $p \in[1, \infty]$, remain constant along the time evolution for regular solutions. In case of $p=1$, we actually have mass conservation. Literature concerning the stability of such solutions is based on these conserved properties. For instance, only spherical polytropes with $-1 / 2 \leqslant v \leqslant 7 / 2$ have finite mass, and if $v<7 / 2$ they also have compact support.

The restriction $\mu<3 / 2$, appearing in [11,13,20,23], was removed in [12,14] by minimizing the energy functional in the mass-Casimir space

$$
\Lambda_{M}^{\mu}=\left\{f: \mathbb{R}^{6} \rightarrow \mathbb{R}_{0}^{+} ; \frac{\mu}{\mu+1}\|f\|_{\mathrm{L}^{1+1 / \mu}\left(\mathbb{R}^{6}\right)}^{1+1 / \mu}+\left(\frac{7}{2}-\mu\right)\|f\|_{\mathrm{L}^{1}\left(\mathbb{R}^{6}\right)}=M\right\} .
$$

Although these works cover the range $0<\mu \leqslant \frac{7}{2}$, these results do not determine exactly neither the spherical polytrope minimizer nor the set of minimizers. In [8] the polytrope corresponding to $\mu=0, c=1, \int_{\mathbb{R}^{3}} v_{0} \mathrm{~d} x=M$ was obtained as the minimizer of

$$
\inf \left\{E(f) ; f \in \mathrm{L}_{+}^{1}\left(\mathbb{R}^{6}, M\right) \cap \mathrm{L}_{+}^{\infty}\left(\mathbb{R}^{6}, 1\right)\right\},
$$

where

$$
\mathrm{L}_{+}^{p}\left(\mathbb{R}^{n}, F\right):=\left\{g \in \mathrm{L}^{p}\left(\mathbb{R}^{n}\right) ; g: \mathbb{R}^{n} \rightarrow \mathbb{R}_{0}^{+},\|g\|_{\mathrm{L}^{p}\left(\mathbb{R}^{n}\right)}=F\right\} .
$$

The dynamical stability criteria developed in $[8,11-14,19,20,23]$ are established in terms of the functionals

$$
d(f, h)=O(f)-O(h)+\frac{1}{8 \pi}\left\|\nabla \phi_{f}-\nabla \phi_{h}\right\|_{\mathrm{L}^{2}\left(\mathbb{R}^{3}\right)}^{2},
$$


where $O$ denotes the functional to minimize, i.e. the Energy-Casimir or Energy functionals, depending on the variational problem under consideration with respect to $\mu$ for functions in the space $\mathrm{L}_{+}^{1}\left(\mathbb{R}^{6}, M\right), \Lambda_{M}^{\mu}$ or $\mathrm{L}_{+}^{1}\left(\mathbb{R}^{6}, M\right) \cap$ $\mathrm{L}_{+}^{\infty}\left(\mathbb{R}^{6}, 1\right)$, respectively. Also, $\phi_{f}$ denotes the solution to the Poisson equation $\Delta_{x} \phi_{f}=4 \pi \rho_{f}$. If $v_{\mu}$ is a minimizer, the dynamical stability criteria establish that for $f^{0}$ as initial condition in the above spaces and "close to" $v_{\mu}$, the solution remains close to the set of minimizers in terms of $d$. Some of these results are valid for a general functional framework and for other families of stationary solutions of the VP system, see [13,14] for more details.

Stability criteria should be determined by fixing three concrete features:

(1) the group of invariants associated with the structure of the system;

(2) the concept of distance to be used;

(3) the notion of admissible perturbation stemming from the functional neighbourhood set involved in the stability criteria.

Let us specify some aspects of the stability criteria in accordance with the previous features.

The solutions of the VP system exhibit some invariance properties up to the noncompact group of symmetries consisting of space translations, which give rise to the notion of orbit of a stationary solution as the more appropriate set of functions which remains close to the time dependent solutions. Since the solutions to the VP system are invariant by space translations, the orbit of the solution $f$ is described by $\left\{f(t, x+k, v) ; k \in \mathbb{R}^{3}\right\}$. The main idea of orbital stability relies on the fact that a stationary solution $f_{S}$ is orbitally stable if small perturbations of $f_{S}$ remain close to the orbit of $f_{S}$ along the evolution (in some sense to be precised in terms of the chosen distance and the admissible perturbations, points (2) and (3)). Furthermore, the orbit set is optimal as shown by the Galilean invariance property: if $f$ is a solution to the VP system, then for all $u \in \mathbb{R}^{3}$ we have that $f^{u}(x, v)=f(x-t u, v-u)$ is also a solution with initial condition $f(x, v-u)$. In the case of polytropes $v_{\mu}$, by choosing $u$ small enough (in norm) we obtain initial conditions close to $v_{\mu}$, meanwhile the corresponding solution only travels close to $\left\{v_{\mu}(t, x+k, v) ; k \in \mathbb{R}^{3}\right\}$. We will use the expression "orbital stability" to invoke any criterium for which the orbit of a stationary solution is the set of functions which remains close to a perturbed VP solution. This concept has been widely employed in other contexts such as bound states and traveling wave solutions to nonlinear PDE's, for instance Klein-Gordon and Schrödinger equations where similar invariances hold (see $[6,7,9])$.

The deduction of orbital stability of a stationary state by variational arguments implies a detailed analysis of the set of minimizers. In $[8,11]$ some orbital stability criteria in terms of the functional $d$ were achieved for the polytropes with $0 \leqslant \mu<\frac{3}{2}$ by considering the Casimir functional. The general result obtained in [14] might be interpreted in our context as follows. Firstly, it is proved [14, Theorem 3] that "up to a shift in $x$-space there are at most two minimizers" in the set of minimizers of the energy with mass-Casimir constraints $\Lambda_{M}^{\mu}$. Then, under the extra assumption of having an isolated minimizer, it is proved [14, Theorem 4(b)] that this is dynamically stable in terms of (1.8) in the massCasimir space $\Lambda_{M}^{\mu}$. In the case that one of these two possible minimizers is a spherical polytrope, there are not provided in [14] the value of the constants $c$ and $E_{0}$ determining (1.4).

After this paper was written, in a personal communication Y. Guo and G. Rein called our attention on the work [21], where the assumption that the minimizer must be isolated in order to obtain stability is removed. In [21] it is proved that for any minimizer obtained in [14] and for any initial condition $f(0) \in C_{0}^{1}\left(\mathbb{R}^{6}\right) \cap \Lambda_{M}^{\mu}$ such that

$$
d\left(f(0), v_{\mu}\right)+\frac{1}{8 \pi}\left\|\nabla \phi_{f(0)}-\nabla \phi_{v_{\mu}}\right\|_{\mathrm{L}^{2}\left(\mathbb{R}^{3}\right)}^{2}<\delta,
$$

there exists a shift vector, $a \in \mathbb{R}^{3}$, such that

$$
d\left(f(t), T^{a} v_{\mu}\right)+\frac{1}{8 \pi}\left\|\nabla \phi_{f(t)}-\nabla \phi_{T^{a} v_{\mu}}\right\|_{\mathrm{L}^{2}\left(\mathbb{R}^{3}\right)}^{2}<\epsilon,
$$

where $T^{a} v_{\mu}(x, v)=v_{\mu}(x+a, v)$.

Concerning the concept of distance it has been shown that the functional $d\left(f, v_{\mu}\right)$ is nonnegative for $f \in \Lambda_{M}^{\mu}$. However, for polytropes (among other solutions) one may think in $d\left(f, v_{\mu}\right)$ as a weighted $L^{2}$-difference between $v_{\mu}$ and $f$ in the following sense: if $1 \leqslant \mu \leqslant \frac{7}{2}$ it can be proved

$$
d\left(f, v_{\mu}\right) \geqslant C\left\|v_{\mu}-f\right\|_{\mathrm{L}^{2}\left(\mathbb{R}^{6}\right)}^{2}, \quad f \in \Lambda_{M}^{\mu} \text { with } f \leqslant f_{\max },
$$


where $f_{\max } \geqslant\left\|v_{\mu}\right\|_{\infty}$ and $C=\frac{2}{\mu} \inf _{0<f \leqslant f_{\max }}\left\{f^{1 / \mu-1}\right\}$, see [14] for more details. An alternative stability criterium that uses perturbative techniques was obtained in [22, pp. 264] in terms of the $\mathrm{L}^{2}\left(\mathbb{R}^{6}\right)$ norm of the distribution, although it only covers the case $\mu=1$.

In [14], the functional neighbourhood defining stability is the mass-Casimir space $\Lambda_{M}^{\mu}$ and the constant $c$ and $E_{0}$ are not a priori determined for the possible polytropic spherical minimizer. In [22], the constraints $\|f\|_{\mathrm{L}^{\infty}} \leqslant M$, $\operatorname{supp}(f) \leqslant M$ and $E(f) \leqslant M$ determine the functional neighbourhood for the orbital stability.

In this work, we determine an orbital stability criterium in a functional neighbourhood of the spherical polytropes with distance function $\mathrm{L}^{1}\left(\mathbb{R}^{6}\right)$, which covers the whole range $0 \leqslant \mu<7 / 2$. We use a different variational argument that involves two constraints and implies the uniqueness of the minimizer. The Plummer case $\mu=7 / 2$ is analyzed in [14].

We deal with the variational problem

$$
I_{M, J}^{\mu}:=\inf \left\{E(f) ; f \in \Gamma_{M, J}^{\mu}\right\}
$$

where $\Gamma_{M, J}^{\mu}=\mathrm{L}_{+}^{1}\left(\mathbb{R}^{6}, M\right) \cap \mathrm{L}_{+}^{1+1 / \mu}\left(\mathbb{R}^{6}, J\right)$. We will prove that the polytropic gas sphere solutions are orbitally stable in the following sense:

Theorem 1.1 (Orbital Stability). Let $\mu \in[0,7 / 2)$ and $\epsilon>0$. Also, let $v_{\mu}$ be a spherical polytropic solution given by (1.4) with $\left\|v_{\mu}\right\|_{\mathrm{L}^{1}\left(\mathbb{R}^{6}\right)}=M$ and $\left\|v_{\mu}\right\|_{\mathrm{L}^{1+1 / \mu}\left(\mathbb{R}^{6}\right)}=J$. Then, there exists $\delta=\delta(\epsilon)>0$ such that for every initial condition $f^{0}$ satisfying

(1) $E\left(f_{0}\right)-E\left(v_{\mu}\right) \leqslant \delta$,

(2) $f_{0} \in \Gamma_{M, J}^{\mu} \cap C_{0}^{1}\left(\mathbb{R}^{6}\right)$,

the associated solution $f$ to (1.1)-(1.3) verifies

$$
\inf _{k \in \mathbb{R}^{3}}\left\|f(t, \cdot, \cdot)-v_{\mu}(\cdot-k, \cdot)\right\|_{\mathrm{L}^{1}\left(\mathbb{R}^{6}\right)} \leqslant \epsilon, \quad \forall t \in(0, \infty) .
$$

If $\mu \neq 0$ we also have

$$
\inf _{k \in \mathbb{R}^{3}}\left\|f(t, \cdot, \cdot)-v_{\mu}(\cdot-k, \cdot)\right\|_{\mathrm{L}^{1+1 / \mu}\left(\mathbb{R}^{6}\right)} \leqslant \epsilon, \quad \forall t \in(0, \infty) .
$$

Theorem 1.1 establishes the concept of orbital stability for kinetic equations. One of the main improvements of this result with respect to previous dynamical criteria for the solutions of the VP system is that the stability for the solutions is established in terms of the $\mathrm{L}^{1}\left(\mathbb{R}^{6}\right)$ norm, which could be the natural in the VP context. This approach allows to cover the range of polytropes with $\mu \in[0,7 / 2)$. Also, the stability criteria are established for any polytrope $v_{\mu}$ in terms of its mass and $\mathrm{L}^{1+1 / \mu}$ norm.

The minimization problems (1.10) present several difficulties. First, the energy is a nonconvex functional. Also, it is invariant by space translations which implies a lack of relative compactness of any minimizing sequence. And, finally, the space proposed for the minimization problem, $\Gamma_{M, J}^{\mu}=\mathrm{L}_{+}^{1}\left(\mathbb{R}^{6}, M\right) \cap \mathrm{L}_{+}^{1+1 / \mu}\left(\mathbb{R}^{6}, J\right)$, has two constraints.

Our minimizing argument is based on overcoming the above difficulties through a series of equivalent reduced problems. Section 2 is devoted to the proof of Theorem 1.1 by using the ideas developed in [7]. It is then shown in the next sections that the minimization problem enters in the framework of [7]. Section 3 explores the relation between the variational problem (1.10) and an equivalent problem for the densities. This equivalence is obtained by finding a series of variational problems whose analysis involves the exact solvability of the intermediate problems. In fact, we prove that beyond this equivalence there is a deeper concept which implies that the minimizing sequences are related in the sense of Theorem 3.1. The relation between the different minimization problems allows to give a new minimizing argument for (1.10), which constitutes the key result of this paper. In Section 4 we minimize the reduced problem obtained in the previous section. The argument developed at this point is related to those used in [19]. Finally, in Appendix A the connection between the minimization problems associated with the Casimir energies, the Energy with mass-Casimir constraint and (1.10) are analyzed. 


\section{Orbital stability: proof of Theorem 1.1}

Several approaches have been developed in other contexts to study orbital stability properties. We first mention that the techniques used in $[9,10]$ to deal with the orbital stability of stationary solutions to nonlinear wave equations are strongly based on the analysis of the linearized operators. These arguments mainly consist in considering an abstract Hamiltonian system which is invariant under a group of operators and then studying the effects of these invariances on the stability of solitary waves. We also refer to [7], where variational techniques valid for Schrödinger-type equations were considered. To prove Theorem 1.1, in this work we follow the argumental scheme of [7] sketched below:

(1) The system of equations is well-posed in a particular functional framework and the solutions satisfy some conservation laws.

(2) Stationary solutions minimize the variational problem

$$
\inf \{O(u) ; u \in X, R(u)=M\},
$$

where the functional $O$ as well as the constraints given by $R$ are conserved quantities of the solutions. We remark that if the functionals $O$ and $R$ are invariant by a noncompact group of symmetries, then the set of minimizers of (2.1) is a noncompact set.

(3) All minimizing sequences for (2.1) are relatively compact up to symmetries. In our case, this means that for any minimizing sequence $f_{n}$, there exists $y_{n} \in \mathbb{R}^{3}$ such that the sequence $f_{n}\left(\cdot-y_{n}, \cdot\right)$ is relatively compact in $L^{1}$.

Let us notice that the concept of solution considered in this work assumes that the sufficient conditions necessary to claim the well-posedness (existence and uniqueness) of the problem hold, see for example [4,18] for a review.

Since assumption (1) is verified by $v_{\mu}, \mu \in[0,7 / 2$ ), we shall focus our attention on proving that (2) and (3) are also satisfied. In this direction we have the following result.

Theorem 2.1. For every $0 \leqslant \mu<7 / 2, M>0$ and $J>0$ there exists a minimum of (1.10). Furthermore, this minimum is reached in the orbit of the spheric polytrope $v_{\mu}$ verifying $\left\|v_{\mu}\right\|_{L^{1}\left(\mathbb{R}^{6}\right)}=M$ and $\left\|v_{\mu}\right\|_{L^{1+1 / \mu}\left(\mathbb{R}^{6}\right)}=J$. More precisely, every minimizing sequence $f_{n}$ is relatively compact in $\mathrm{L}^{1}\left(\mathbb{R}^{6}\right)$ up to spatial translations, i.e., there exists $y_{n} \in \mathbb{R}^{3}$ such that $f_{n}\left(\cdot-y_{n}, \cdot\right)$ is relatively compact in $\mathrm{L}^{1}\left(\mathbb{R}^{6}\right)$. In the case $\mu \in(0,7 / 2), f_{n}\left(\cdot-y_{n}, \cdot\right)$ is also relatively compact in $\mathrm{L}^{1+1 / \mu}\left(\mathbb{R}^{6}\right)$.

Once we have proved that (1)-(3) holds, it is a simple matter to show that the solutions $v_{\mu}$ are orbitally stable.

Proof of Theorem 1.1. If the thesis of Theorem 1.1 is not true, there would exist $\epsilon_{0}, f_{n}^{0}$ and $t_{n}$ such that:

$$
\left\{\begin{array}{l}
f_{n}^{0} \in \Gamma_{M, J}^{\mu} \cap C_{0}^{1}\left(\mathbb{R}^{6}\right), \\
E\left(f_{n}^{0}\right) \rightarrow E\left(v_{\mu}\right), \quad n \rightarrow \infty, \\
\forall k \in \mathbb{R}^{3}\left\{\begin{array}{l}
\left\|f_{n}\left(t_{n}, \cdot, \cdot\right)-v_{\mu}(\cdot-k, \cdot)\right\|_{\mathrm{L}^{1}\left(\mathbb{R}^{6}\right)}>\epsilon_{0} \\
\text { or } \\
\left\|f_{n}\left(t_{n}, \cdot, \cdot\right)-v_{\mu}(\cdot-k, \cdot)\right\|_{\mathrm{L}^{1+1 / \mu}\left(\mathbb{R}^{6}\right)}>\epsilon_{0},
\end{array}\right.
\end{array}\right.
$$

for $\mu \in(0,7 / 2)$, and

$$
\left\{\begin{array}{l}
f_{n}^{0} \in \Gamma_{M, J}^{0}, \\
E\left(f_{n}^{0}\right) \rightarrow E\left(v_{0}\right), \quad n \rightarrow \infty, \\
\forall k \in \mathbb{R}^{3}\left\|f_{n}\left(t_{n}, \cdot, \cdot\right)-v_{0}(\cdot-k, \cdot)\right\|_{\mathrm{L}^{1}\left(\mathbb{R}^{6}\right)}>\epsilon_{0},
\end{array}\right.
$$

for $\mu=0$. Now, we use the conservation of the mass and of the $\mathrm{L}^{p}$ norms to deduce that $\left\{f_{n}\left(t_{n}, \cdot, \cdot\right)\right\}$ is a minimizer for (1.10), since

$$
f_{n}\left(t_{n}, \cdot, \cdot\right) \in \Gamma_{M, J}^{\mu}, \quad E\left(f_{n}^{0}(\cdot, \cdot)\right)=E\left(f_{n}\left(t_{n}, \cdot, \cdot\right)\right) \rightarrow E\left(v_{\mu}\right) .
$$

Then, (2.2) or (2.3) are clearly in contradiction with Theorem 2.1 as we can extract a subsequence from $\left\{f_{n}\left(t_{n}, \cdot, \cdot\right)\right\}$ which is relatively compact up to translations. 
The rest of the paper is devoted to prove Theorem 2.1.

\section{On an equivalent reduced problem}

As we pointed out in the introduction, we now propose a variational approach related to the problems stated in (1.10) in order to prove the orbital stability of spherical polytropes with $0 \leqslant \mu<7 / 2$. We actually show the relative compactness in $\mathrm{L}^{p}$ of any minimizing sequence and that the minimum value is achieved in a particular polytropic solution (up to translations in both cases). The method proposed in this work tries to minimize the difficulties by considering a sequence of equivalent reduced problems. The equivalence relations rely on the fact that between the functions $f \in \Gamma_{M, J}^{\mu}$ with the same density $\rho(x)=\int_{\mathbb{R}^{3}} f(x, v) \mathrm{d} v$ there are special functions $\tilde{f}$ whose energy is as small as possible. Besides, $\tilde{f}$ can be expressed in terms of $\rho$ and $J$ by

$$
\tilde{f}(x, v)=J\left(\frac{1}{2}\left(\frac{3 \rho(x)}{4 \pi J}\right)^{\frac{2}{3}}-\frac{|v|^{2}}{2}\right)_{+}^{0} \equiv \begin{cases}J & \text { if }|v| \leqslant\left(\frac{3 \rho(x)}{4 \pi J}\right)^{\frac{1}{3}}, \\ 0 & \text { elsewhere }\end{cases}
$$

when $\mu=0$, and

$$
\tilde{f}(x, v)=\left(\left(\frac{2 \mu+5}{2(\mu+1)} C \rho^{\frac{2}{2 \mu+3}}(x)-\frac{3}{2(\mu+1)} C^{\frac{2 \mu+3}{3}} \frac{1}{K_{1,1}} \frac{|v|^{2}}{2}\right)_{+}\right)^{\mu}
$$

for $\mu>0$. Here,

$$
C=\frac{J^{1+1 / \mu}}{\int_{\mathbb{R}^{3}} \rho(x)^{\frac{2 \mu+5}{2 \mu+3}} \mathrm{~d} x}
$$

and $K_{1,1}$ is a positive constant (to be determined) depending only on $\mu$. This definition leads to

$$
E_{\mathrm{KIN}}(\tilde{f})=\frac{3^{\frac{5}{3}}}{10(4 \pi J)^{\frac{2}{3}}} \int_{\mathbb{R}^{3}} \rho(x)^{\frac{5}{3}} \mathrm{~d} x,
$$

for $\mu=0$, and

$$
\begin{aligned}
& \int_{\mathbb{R}^{3}} \tilde{f}^{1+1 / \mu}(x, v) \mathrm{d} v=C \rho(x)^{\frac{2 \mu+5}{2 \mu+3}}, \\
& E_{\mathrm{KIN}}(\tilde{f})=\frac{\left(\int_{\mathbb{R}^{3}} \rho(x)^{\frac{2 \mu+5}{2 \mu+3}} \mathrm{~d} x\right)^{\frac{2 \mu+3}{3}}}{J^{\frac{2(\mu+1)}{3}}} K_{1,1},
\end{aligned}
$$

for $\mu \in(0,7 / 2)$. Let us observe that the integrals involved in (3.3) and (3.4) are well defined in virtue of the following inequality

$$
\int_{\mathbb{R}^{3}}|\rho|^{\frac{2 \mu+5}{2 \mu+3}} \mathrm{~d} x \leqslant C\|f\|_{\mathrm{L}^{1+1 / \mu}\left(\mathbb{R}^{6}\right)}^{\frac{2 \mu+2}{2 \mu+3}}\left(\int_{\mathbb{R}^{6}}|v|^{2}|f(x, v)| \mathrm{d} v \mathrm{~d} x\right)^{\frac{3}{2 \mu+3}},
$$

which can be proved by well known arguments based on the nonnegativity of $f$. Thus, the total energy is given by

$$
E(\tilde{f})=E_{J}^{\mu}(\rho):=K \frac{\left(\int_{\mathbb{R}^{3}} \rho(x)^{\frac{2 \mu+5}{2 \mu+3}} \mathrm{~d} x\right)^{\frac{2 \mu+3}{3}}}{J^{\frac{2(\mu+1)}{3}}}-\frac{1}{2} \int_{\mathbb{R}^{6}} \frac{\rho(x) \rho(y)}{|x-y|} \mathrm{d} x \mathrm{~d} y,
$$

where $K=3^{5 / 3} /\left(10(4 \pi)^{2 / 3}\right)$ for $\mu=0$ and $K=K_{1,1}$ for $\mu>0$. We can now establish the equivalence between (1.10) and a reduced problem for the densities. The idea of finding an equivalent problem was firstly introduced and solved by G. Rein in [19] in the context of the Energy-Casimir minimization problem. Our argument is summarized in the next theorem. 
Theorem 3.1. Let $M>0, J>0$ and $\mu \in\left[0, \frac{7}{2}\right)$. Consider $f \in \Gamma_{M, J}^{\mu}$ such that $E(f)<\infty$. Then, there exists a positive function $\tilde{f}$, defined by (3.1) or (3.2) in terms of $\rho$ and $J$, verifying

(1) $f$ and $\tilde{f}$ have the same mass density $\rho$,

(2) $\|f\|_{\mathrm{L}^{1+1 / \mu}\left(\mathbb{R}^{6}\right)}=\|\tilde{f}\|_{\mathrm{L}^{1+1 / \mu}\left(\mathbb{R}^{6}\right)}=J$,

(3) $E(\tilde{f}) \leqslant E(f)$.

In consequence the variational problems

$$
R_{J, M}^{\mu}:=\inf \left\{E_{J}^{\mu}(\rho) ; \rho \in \mathrm{L}_{+}^{1}\left(\mathbb{R}^{3}, M\right) \cap \mathrm{L}^{\frac{2 \mu+5}{2 \mu+3}}\left(\mathbb{R}^{3}\right)\right\}
$$

and (1.10) are equivalent in the following sense:

(a) Their infima values coincide: $R_{J, M}^{\mu}=I_{J, M}^{\mu}$.

(b) Let $\left\{f_{n}\right\}$ be a minimizing sequence for any problem (1.10). Then, $\rho_{n}(x)=\int_{\mathbb{R}^{3}} f_{n}(x, v) \mathrm{d} v$ is a minimizing sequence for the corresponding problem (3.6). Moreover, if $\rho_{n}$ is a minimizing sequence for any problem (3.6), then the sequence of functions $\tilde{f}_{n}$ defined by (3.1) or (3.2) with associated densities $\rho_{n}$ is a minimizing sequence for the corresponding problem (1.10).

(c) (1.10) has a minimum if and only if (3.6) also has a minimum. In that case, the corresponding minimizers verify (b).

Remark 3.2. Theorem 3.1 admits a reverse reading. Let us consider a function $\rho \in \mathrm{L}_{+}^{1}\left(\mathbb{R}^{3}, M\right) \cap \mathrm{L}^{\frac{2 \mu+5}{2 \mu+3}}\left(\mathbb{R}^{3}\right)$. Then, the function $\tilde{f}$ defined by (3.1) or (3.2), with associated density function $\rho$, satisfies:

(1) $\tilde{f} \in \mathrm{L}_{+}^{1}\left(\mathbb{R}^{6}, M\right) \cap \mathrm{L}_{+}^{1+1 / \mu}\left(\mathbb{R}^{6}, J\right)$,

(2) $\tilde{f}$ verifies (3.3), (3.4),

(3) $E(\tilde{f}) \leqslant E(f), \forall f \in \mathrm{L}_{+}^{1+1 / \mu}\left(\mathbb{R}^{6}, J\right)$ such that $\int_{\mathbb{R}^{3}} f \mathrm{~d} v=\int_{\mathbb{R}^{3}} \tilde{f} \mathrm{~d} v=\rho$.

Theorem 3.1 requires the study of an auxiliary problem, defined in the following

Lemma 3.3. The minimization problem

$$
K_{G, H}=\inf \left\{\frac{1}{2} \int_{\mathbb{R}^{3}}|v|^{2} z(v) \mathrm{d} v ; z \in \mathrm{L}_{+}^{1}\left(\mathbb{R}^{3}, G\right) \cap \mathrm{L}_{+}^{1+1 / \mu}\left(\mathbb{R}^{3}, H\right)\right\}
$$

has a minimum for $0 \leqslant \mu<7 / 2$. $K_{G, H}$ verifies

$$
K_{G, H}=\frac{G^{\frac{2 \mu+5}{3}}}{H^{\frac{2 \mu+2}{3}}} K_{1,1}, \quad \text { for } \mu>0
$$

and

$$
K_{G, H}=\frac{(3 G)^{\frac{5}{3}}}{10(4 \pi H)^{\frac{2}{3}}}, \quad \text { for } \mu=0,
$$

where $K_{1,1}$ is defined by (3.7) with $G=H=1$. This minimum is reached by a unique function $z_{G, H}$. If $\mu>0$ and $G, H>0$, then $z_{G, H}$ is explicitly given by

$$
z_{G, H}(v)=\left(\left(\frac{2 \mu+5}{2(\mu+1)} \frac{H^{\frac{\mu+1}{\mu}}}{G}-\frac{3}{2(\mu+1)} \frac{H^{\frac{\mu+1}{\mu} \frac{2 \mu+3}{3}}}{G^{\frac{2 \mu+5}{3}}} \frac{1}{K_{1,1}} \frac{|v|^{2}}{2}\right)_{+}\right)^{\mu} .
$$

In the case $\mu=0, z_{G, H}$ is defined by

$$
z_{G, H}(v)= \begin{cases}H & \text { if }|v| \leqslant\left(\frac{3 G}{4 \pi H}\right)^{1 / 3} \\ 0 & \text { elsewhere. }\end{cases}
$$


If $G=H=0$, (3.7) trivially implies $K_{0,0}=0$ and $z_{0,0} \equiv 0$.

The rest of this section is devoted to prove the last two results.

Proof of Theorem 3.1. The main idea of the proof is to analyze a chain of equivalent variational problems starting with $(1.10) \equiv\left(\mathrm{P}_{1}\right)$ and finishing with $(3.6) \equiv\left(\mathrm{P}_{4}\right)$ :

$$
\begin{aligned}
& \left(\mathrm{P}_{1}\right) \quad \inf _{f}\left\{E(f) ; f \in \mathrm{L}_{+}^{1}\left(\mathbb{R}^{6}, M\right) \cap \mathrm{L}_{+}^{1+1 / \mu}\left(\mathbb{R}^{6}, J\right)\right\}, \\
& \left(\mathrm{P}_{2}\right) \inf _{\rho \in \mathrm{L}_{+}^{1}\left(\mathbb{R}^{3}, M\right)}\left\{\inf _{\substack{h \in \mathrm{L}_{+}^{1}\left(\mathbb{R}^{3}, J^{1+1 / \mu}\right) \\
\operatorname{Supp}(h)=\operatorname{Supp}(\rho)}}\left\{\inf _{\int f^{1+1 / \mu} \mathrm{d} v=h}\left\{E_{\mathrm{KIN}}(f)\right\}\right\}+E_{\mathrm{POT}}(\rho)\right\} \text {, } \\
& \text { ( } \left.\mathrm{P}_{3}\right) \inf _{\rho \in \mathrm{L}_{+}^{1}\left(\mathbb{R}^{3}, M\right)}\left\{\inf _{\substack{h \in \mathrm{L}_{+}^{1}\left(\mathbb{R}^{3}, J^{1+1 / \mu}\right) \\
\operatorname{Supp}(h)=\operatorname{Supp}(\rho)}}\left\{\int K \frac{\rho^{\frac{2 \mu+3}{3}}}{h^{\frac{2 \mu}{3}}} \mathrm{~d} x\right\}+E_{\mathrm{POT}}(\rho)\right\} \text {, } \\
& \text { ( } \left.\mathrm{P}_{4}\right) \inf _{\rho \in \mathrm{L}_{+}^{1}\left(\mathbb{R}^{3}, M\right)}\left\{\frac{K}{J^{\frac{2 \mu+2}{3}}}\left(\int \rho^{\frac{2 \mu+5}{2 \mu+3}} \mathrm{~d} x\right)^{\frac{2 \mu+3}{3}}+E_{\mathrm{POT}}(\rho)\right\} .
\end{aligned}
$$

However, to control the densities of any minimizing sequence we have to deal with space pointwise versions of these problems. It is also necessary a detailed analysis of the intermediate problems $\left(\mathrm{P}_{2}\right)$ and $\left(\mathrm{P}_{3}\right)$ which allows to describe the intrinsic deep relation between the minimizing sequences given by properties (1), (2) and (3) in Theorem 3.1. For the sake of simplicity we split the proof in several steps.

Step 1: Problem $\left(P_{2}\right)$. A function $\tilde{f}$ verifying (1), (2) and (3) in Theorem 3.1 minimizes the problem

$$
\inf \{E(l) ; l \in \Theta\} \text {, }
$$

where $\Theta=\left\{l \in \mathrm{L}_{+}^{1+1 / \mu}\left(\mathbb{R}^{6}, J\right), \int_{\mathbb{R}^{3}} l(x, v) \mathrm{d} v=\rho(x)\right.$ a.e. $\left.x \in \mathbb{R}^{3}\right\}$. This problem is equivalent to study

$$
\inf \left\{E_{\mathrm{KIN}}(l) ; l \in \Theta\right\}
$$

because the potential energy only depends on the function $\rho$.

Let us first consider the case $\mu>0$. We define the sets

$$
\Theta_{\rho, h}=\left\{l: \mathbb{R}^{6} \rightarrow \mathbb{R}_{0}^{+} ; \int_{\mathbb{R}^{3}} l \mathrm{~d} v=\rho(x), \int_{\mathbb{R}^{3}} l^{1+1 / \mu} \mathrm{d} v=h(x), \text { a.e. } x \in \mathbb{R}^{3}\right\}
$$

and

$$
\Theta_{\rho}=\left\{h \in \mathrm{L}_{+}^{1}\left(\mathbb{R}^{3}, J^{1+1 / \mu}\right) ; \operatorname{Supp}(\rho)=\operatorname{Supp}(h)\right\} .
$$

Then, $\Theta=\bigcup_{h \in \Theta_{\rho}} \Theta_{\rho, h}$. This simple idea provides the equivalence between (3.10) and the problem

$$
\inf \left\{\inf \left\{E_{\mathrm{KIN}}(l) ; l \in \Theta_{\rho, h}\right\} ; h \in \Theta_{\rho}\right\} .
$$

In order to solve (3.11) we first analyze

$$
\inf \left\{E_{\mathrm{KIN}}(l) ; l \in \Theta_{\rho, h}\right\}
$$

for any fixed but arbitrary $h \in \Theta_{\rho}$. The constraints defining $\Theta_{\rho, h}$ are fixed for any $x \in \mathbb{R}^{3}$. Then, we propose to study (3.12) by considering the problem

$$
P_{x}=\inf \left\{\frac{1}{2} \int_{\mathbb{R}^{3}}|v|^{2} l(x, v) \mathrm{d} v ; l \geqslant 0, \int_{\mathbb{R}^{3}} l(x, v) \mathrm{d} v=\rho(x), \int_{\mathbb{R}^{3}} l(x, v)^{1+1 / \mu} \mathrm{d} v=h(x)\right\}
$$

for almost everywhere $x \in \mathbb{R}^{3}$ fixed but arbitrary (this is the spatial pointwise version of the inner problem considered in $\left(\mathrm{P}_{2}\right)$ ). Our argument is founded on the following basic idea: If (3.13) has a minimum $P_{x}$ a.e. $x \in \mathbb{R}^{3}$ and this 
minimum is achieved by a function $l_{x}(v)$, then (3.12) has also a minimum $\int_{\mathbb{R}^{3}} P_{x} \mathrm{~d} x$ and it is achieved by the function $l(x, v)=l_{x}(v)$.

We can control the dependence with respect to $x$ in (3.13) because this problem obeys the general profile of (3.7), where we identify $l(x, \cdot)=z(\cdot), G=\rho(x)$ and $H^{1+1 / \mu}=h(x)$. Let us observe that the dependence with respect to $x$ is entirely concentrated on the value of the constants $G$ and $H$. Lemma 3.3 gives the existence of a minimizer for (3.13), where $z_{G, H}$ depends on $G$ and $H$. The detailed analysis of this dependence done in the proof of Lemma 3.3 is motivated by the fact that we have to define $h_{x}(\cdot)=z_{G, H}(\cdot)$ for $G=\rho(x)$ and $H^{1+1 / \mu}=h(x)$. Therefore, we have analogous results for the problem (3.13): If $x \in \operatorname{Supp}(\rho)=\operatorname{Supp}(h)$, then (3.13) has a minimum

$$
P_{x}=\frac{\rho(x)^{\frac{2 \mu+5}{3}}}{h(x)^{\frac{2 \mu}{3}}} K_{1,1} .
$$

This minimum is achieved by

$$
l(x, v)=l_{x}(v)=\left(\left(\frac{2 \mu+5}{2(\mu+1)} \frac{h(x)}{\rho(x)}-\frac{3}{2(\mu+1)} \frac{h(x)^{\frac{2 \mu+3}{3}}}{\rho(x)^{\frac{2 \mu+5}{3}}} \frac{1}{K_{1,1}} \frac{|v|^{2}}{2}\right)_{+}\right)^{\mu} .
$$

This concludes the analysis of problem $\left(\mathrm{P}_{2}\right)$.

Step 2: Problem $\left(P_{3}\right)$. If $x \in \mathbb{R}^{3}-\operatorname{Supp}(\rho)$, then $P_{x}=0$. Now, using (3.14), we can rewrite (3.11) as

$$
\inf \left\{\int_{\mathbb{R}^{3}} \frac{\rho(x)^{\frac{2 \mu+5}{3}}}{h(x)^{\frac{2 \mu}{3}}} K_{1,1} \mathrm{~d} x ; h \in \Theta_{\rho}\right\}
$$

which is directly solvable by using Hölder's inequality in the following way

$$
\int_{\mathbb{R}^{3}} \rho(x)^{\frac{2 \mu+5}{2 \mu+3}} \mathrm{~d} x \leqslant\left(\int_{\mathbb{R}^{3}} \frac{\rho(x)^{\frac{2 \mu+5}{3}}}{h(x)^{\frac{2 \mu}{3}}} \mathrm{~d} x\right)^{\frac{3}{2 \mu+3}}\left(\int_{\mathbb{R}^{3}} h(x) \mathrm{d} x\right)^{\frac{2 \mu}{2 \mu+3}},
$$

or equivalently

$$
\frac{\left(\int_{\mathbb{R}^{3}} \rho(x)^{\frac{2 \mu+5}{2 \mu+3}} \mathrm{~d} x\right)^{\frac{2 \mu+3}{3}}}{\left(\int_{\mathbb{R}^{3}} h(x) \mathrm{d} x\right)^{\frac{2 \mu}{3}}} \leqslant \int_{\mathbb{R}^{3}} \frac{\rho(x)^{\frac{2 \mu+5}{3}}}{h(x)^{\frac{2 \mu}{3}}} \mathrm{~d} x .
$$

Furthermore, the equality holds if and only if $h$ is proportional to $\rho^{\frac{2 \mu+5}{2 \mu+3}}$. As consequence, the mimimun of (3.15) is

$$
\frac{\left(\int_{\mathbb{R}^{3}} \rho(x)^{\frac{2 \mu+5}{2 \mu+3}} \mathrm{~d} x\right)^{\frac{2 \mu+3}{3}}}{J^{\frac{2(\mu+1)}{3}}} K_{1,1}
$$

and it is reached when $h=C \rho^{\frac{2 \mu+5}{2 \mu+3}}$, where

$$
C=\frac{J^{1+1 / \mu}}{\int_{\mathbb{R}^{3}} \rho(x)^{\frac{2 \mu+5}{2 \mu+3}} \mathrm{~d} x} .
$$

We conclude that the infimum of (3.10) is achieved by $\tilde{f}$, defined as

$$
\tilde{f}(x, v)=\left(\left(\frac{2 \mu+5}{2(\mu+1)} C \rho^{\frac{2}{2 \mu+3}}-\frac{3}{2(\mu+1)} C^{\frac{2 \mu+3}{3}} \frac{1}{K_{1,1}} \frac{|v|^{2}}{2}\right)_{+}\right)^{\mu},
$$

and the infimum value is

$$
E_{\mathrm{KIN}}(\tilde{f})=\frac{\left(\int_{\mathbb{R}^{3}} \rho(x)^{\frac{2 \mu+5}{2 \mu+3}} \mathrm{~d} x\right)^{\frac{2 \mu+3}{3}}}{J^{\frac{2(\mu+1)}{3}}} K_{1,1} .
$$


In the case $\mu=0$ our argument becomes easier because both conditions defining $\Theta$ are local in space. By using the previous arguments we deduce in this case

$$
\tilde{f}(x, v)=l_{x}(v)=J\left(\frac{1}{2}\left(\frac{3 \rho(x)}{4 \pi J}\right)^{\frac{2}{3}}-\frac{|v|^{2}}{2}\right)_{+}^{0}
$$

and

$$
E_{\mathrm{KIN}}(\tilde{f})=\frac{3^{\frac{5}{3}}}{10(4 \pi J)^{\frac{2}{3}}} \int_{\mathbb{R}^{3}} \rho(x)^{\frac{5}{3}} \mathrm{~d} x,
$$

which concludes the proof of Theorem 3.1.

We now prove Lemma 3.3.

Proof of Lemma 3.3. Set $S_{\mu}(z)=\frac{1}{2} \int_{\mathbb{R}^{3}}|v|^{2} z(v) \mathrm{d} v$ and consider $K_{G, H}$ defined by (3.7).

The positivity of $K_{G, H}$ is deduced from the inequality

$$
\|g\|_{\mathrm{L}^{1}\left(\mathbb{R}^{3}\right)}^{\frac{2 \mu+5}{3}} \leqslant C\|g\|_{\mathrm{L}^{1+1 / \mu}\left(\mathbb{R}^{3}\right)}^{\frac{2 \mu+2}{3}}|x|_{\mathbb{R}^{3}}^{2} g(x) \mathrm{d} x,
$$

which holds for any positive function $g$. By using the scaling $\breve{z}(x)=a z(b x)$, where $a=H^{\mu+1} / G^{\mu}$ and $b=$ $(H / G)^{\frac{\mu+1}{3}}$, we find

$$
K_{G, H}=\frac{G^{\frac{2 \mu+5}{3}}}{H^{\frac{2 \mu+2}{3}}} K_{1,1} .
$$

Also, the minimizers for a pair $G=1, H=1$ are related to the minimizers for $G^{\prime}, H^{\prime}$ by the same scaling. Thus, we can rewrite (3.7) as

$$
\inf \left\{\frac{1}{2} \int_{\mathbb{R}^{3}}|v|^{2} z(v) \mathrm{d} v ; z \in \mathrm{L}_{+}^{1}\left(\mathbb{R}^{3}, G\right),\|z\|_{\mathrm{L}^{1+1 / \mu}\left(\mathbb{R}^{3}\right)} \leqslant H\right\}
$$

since both problems have the same minimum and minimizers (any minimizer $z_{G, H}$ for (3.16) verifies $\left.\left\|z_{G, H}\right\|_{\mathrm{L}^{1+1 / \mu}\left(\mathbb{R}^{3}\right)}=H\right)$.

Let $\left\{z_{n}\right\}$ be a minimizing sequence for (3.16). We have that $\left\|z_{n}\right\|_{\mathrm{L}^{1+1 / \mu}\left(\mathbb{R}^{3}\right)}$ and $\int_{\mathbb{R}^{3}}|v|^{2} z_{n}(v) \mathrm{d} v$ are uniformly bounded. Therefore, $\left\{z_{n}\right\}$ is under the hypotheses of the Dunford-Pettis theorem:

(1) is bounded in $\mathrm{L}^{1}\left(\mathbb{R}^{3}\right)$, because $\left\|z_{n}\right\|_{\mathrm{L}^{1}\left(\mathbb{R}^{3}\right)}=G$;

(2) there is no concentration in any measurable set $A$, since

$$
\int_{A} z_{n}(v) \mathrm{d} v \leqslant\left\|z_{n}\right\|_{\mathrm{L}^{1+1 / \mu}\left(\mathbb{R}^{3}\right)}|A|^{\mu+1} ;
$$

(3) and there is no vanishing, since $\int_{|v| \geqslant R} z_{n}(v) \mathrm{d} v \leqslant \frac{1}{R^{2}} \int_{\mathbb{R}^{3}}|v|^{2} z_{n}(v) \mathrm{d} v$.

Thus, we can extract a subsequence verifying $z_{n} \rightarrow z_{G, H}$ weakly in $\mathrm{L}^{1}\left(\mathbb{R}^{3}\right)$ and

$$
\begin{cases}z_{n} \rightarrow z_{G, H} \text { weakly in } \mathrm{L}^{1+1 / \mu}\left(\mathbb{R}^{3}\right), & \text { for } \mu \in(0,7 / 2), \\ z_{n} \rightarrow z_{G, H} \text { in the weak-* } \mathrm{L}^{\infty}\left(\mathbb{R}^{3}\right) \text { topology, } & \text { for } \mu=0 .\end{cases}
$$

Also $z_{G, H} \in \mathrm{L}^{1}\left(\mathbb{R}^{3}\right) \cap \mathrm{L}^{1+1 / \mu}\left(\mathbb{R}^{3}\right)$ and is nonnegative because it is obtained as a weak limit of nonnegative functions. Using the inequalities 


$$
\begin{aligned}
& \int_{\mathbb{R}^{3}}|v|^{2} z_{G, H}(v) \mathrm{d} v=\lim _{R \rightarrow \infty} \int_{|v| \leqslant R}|v|^{2} z_{G, H}(v) \mathrm{d} v=\lim _{R \rightarrow \infty} \lim _{n \rightarrow \infty} \int_{|v| \leqslant R}|v|^{2} z_{n}(v) \mathrm{d} v \\
& \leqslant \lim _{R \rightarrow \infty} \lim _{n \rightarrow \infty} \int_{\mathbb{R}^{3}}|v|^{2} z_{n}(v) \mathrm{d} v=\lim _{n \rightarrow \infty} \int_{\mathbb{R}^{3}}|v|^{2} z_{n}(v) \mathrm{d} v, \\
& \left\|z_{G, H}\right\|_{\mathrm{L}^{1+1 / \mu}\left(\mathbb{R}^{3}\right)} \leqslant \liminf _{n \rightarrow \infty}\left\|z_{n}\right\|_{\mathrm{L}^{1+1 / \mu}\left(\mathbb{R}^{3}\right)} \leqslant H
\end{aligned}
$$

and

$$
\left\|z_{G, H}\right\|_{\mathrm{L}^{1}\left(\mathbb{R}^{3}\right)} \leqslant \liminf _{n \rightarrow \infty}\left\|z_{n}\right\|_{\mathrm{L}^{1}\left(\mathbb{R}^{3}\right)}=G
$$

we have that $z_{G, H}$ verifies $S_{\mu}\left(z_{G, H}\right) \leqslant \liminf _{n \rightarrow \infty} S_{\mu}\left(z_{n}\right)=K_{G, H}$. To conclude that $z_{G, H}$ is a minimizer for (3.16), we have to check that $\left\|z_{G}\right\|_{\mathrm{L}^{1}\left(\mathbb{R}^{3}\right)}=G$. Let $\epsilon$ be a positive constant. Then, there exist $R$ (depending only on $\epsilon$ ) and $B(R)$ a ball centered in 0 with radius $R$ such that

$$
\begin{aligned}
\int_{B(R)} z_{G, H} & =\lim _{n \rightarrow \infty} \int_{B(R)} z_{n}=G-\lim _{n \rightarrow \infty} \int_{\mathbb{R}^{3}-B(R)} z_{n} \\
& \geqslant G-\lim _{n \rightarrow \infty} \frac{1}{R^{2}} \int_{\mathbb{R}^{3}}|v|^{2} z_{n} \geqslant G-\epsilon .
\end{aligned}
$$

Thus, by using (3.17) and (3.18) we deduce that $\left\|z_{G, H}\right\|_{\mathrm{L}^{1}\left(\mathbb{R}^{3}\right)}=G$.

Once we know that a minimum of (3.16) (equivalent of (3.7)) exists, we study some of the properties of the minimizers. Let us prove that $z_{G, H}$ is a nonincreasing function. For radial nonincreasing rearrangements $z^{*}$ of $z$ (see [17]) we have

$$
\int_{\mathbb{R}^{3}}|v|^{2} z^{*}(v) \mathrm{d} v \leqslant \int_{\mathbb{R}^{3}}|v|^{2} z(v) \mathrm{d} v,
$$

with strict inequality unless $z \equiv z^{*}$. Then, $z_{G, H}$ coincides with its rearranged function and consequently it is a symmetric nonincreasing function.

The expression of these functions is obtained from the Euler-Lagrange equation, which for $\mu>0$ reads

$$
\frac{1}{2}|v|^{2}+\lambda z_{G, H}^{1 / \mu}+\beta \chi=\gamma,
$$

where $\lambda, \beta, \gamma$ are the Lagrange multipliers and the function $\chi$ is defined by

$$
\chi(v)= \begin{cases}0 & \text { if } z_{G, H}(v)>0 \\ \geqslant 0 & \text { if } z_{G, H}(v)=0 .\end{cases}
$$

We first note that $\lambda \neq 0$ since otherwise $\operatorname{Supp}\left(z_{G, H}\right) \subset\left\{v \in \mathbb{R}^{3} ; \frac{1}{2}|v|^{2}=\gamma\right\}$, which is a set of null measure. Then, we have

$$
z_{G, H}(v)=\frac{1}{\lambda^{\mu}}\left(\gamma-\frac{1}{2}|v|^{2}\right)^{\mu}, \quad \text { for } v \in \operatorname{Supp}\left(z_{G, H}\right) .
$$

This expression combined with the nonincreasing and nonnegative character of $z_{G, H}$ implies that $\lambda>0$ and $\operatorname{Supp}\left(z_{G, H}\right) \subset\left\{v \in \mathbb{R}^{3} ; \frac{1}{2}|v|^{2} \leqslant \gamma\right\}$. On the other hand, we have

$$
\gamma-\frac{1}{2}|v|^{2}=\beta \chi(v), \quad \text { for } v \in \mathbb{R}^{3}-\operatorname{Supp}\left(z_{G, H}\right) .
$$

Then, $\beta<0$ (since $z_{G, H}$ is nonincreasing) and

$$
z_{G, H}(v)=\frac{1}{\lambda^{\mu}}\left(\gamma-\frac{1}{2}|v|^{2}\right)_{+}^{\mu}
$$


By using (3.20) we can compute $\lambda$ and $\gamma$. Multiplying (3.19) by $z_{G, H}$ and integrating over $\mathbb{R}^{3}$ we find

$$
K_{G, H}+\lambda H^{1+1 / \mu}=\gamma G \text {. }
$$

The use of radial coordinates leads to the following equality

$$
\begin{aligned}
K_{G, H} & =\frac{1}{2} \int_{\mathbb{R}^{3}}|v|^{2} z_{G, H}(v) \mathrm{d} v=\frac{1}{2} \frac{4 \pi}{\lambda^{\mu}} \int_{0}^{\sqrt{2 \gamma}} r^{4}\left(\beta-\frac{1}{2} r^{2}\right)^{\mu} \mathrm{d} r \\
& =\frac{1}{2} \frac{4 \pi}{\lambda^{\mu}} \frac{3}{\mu+1} \int_{0}^{\sqrt{2 \gamma}} r^{2}\left(\beta-\frac{1}{2} r^{2}\right)^{\mu+1} \mathrm{~d} r=\frac{1}{2} \frac{3 \lambda}{\mu+1} \int_{\mathbb{R}^{3}} z_{G, H}^{1+1 / \mu} \mathrm{d} v \\
& =\frac{3 \lambda}{2(\mu+1)} H^{1+1 / \mu} .
\end{aligned}
$$

From these equations and Lemma 3.3 we have

$$
\lambda=\frac{2(\mu+1)}{3} G^{\frac{2 \mu+5}{3}} H^{-(\mu+1)\left(\frac{1}{\mu}+\frac{2}{3}\right)} K_{1,1} \quad \text { and } \quad \gamma=\frac{2 \mu+5}{3} G^{\frac{2 \mu+2}{3}} H^{-\frac{(2 \mu+2)}{3}} K_{1,1},
$$

which proves (3.8).

In the case $\mu=0$ the corresponding Euler-Lagrange equation reads

$$
\frac{1}{2}|v|^{2}+\lambda \varphi+\beta \chi=\gamma,
$$

where $\lambda, \beta, \gamma$ are the Lagrange multipliers and the functions $\varphi$ and $\chi$ are defined by

$$
\varphi(v)=\left\{\begin{array}{ll}
0 & \text { if } z_{G, H}(v)<H, \\
\geqslant 0 & \text { if } z_{G, H}(v)=H,
\end{array} \quad \chi(v)= \begin{cases}0 & \text { if } z_{G, H}(v)>0 \\
\geqslant 0 & \text { if } z_{G, H}(v)=0 .\end{cases}\right.
$$

Then, (3.21) implies that $\left\{v \in \mathbb{R}^{3} ; 0<z_{G, H}(v)<H\right\}$ has null measure in $\mathbb{R}^{3}$, therefore $z_{G, H}=H$ a.e. $\operatorname{Supp}\left(z_{G, H}\right)$. We finally need to determine $\operatorname{Supp}\left(z_{G}\right)$ in order to give an explicit expression for this function. The symmetric nonincreasing character of $z_{G, H}$ determines that $\operatorname{Supp}\left(z_{G, H}\right)$ must coincide with the ball in $\mathbb{R}^{3}$ with radius $(3 G /(4 \pi H))^{1 / 3}$, concluding (3.9).

\section{Analysis of the reduced problem}

In this section we study the minimization problem (3.6). We adapt the techniques employed in [19] to deal with reduced equivalent problems to those concerning the Energy-Casimir functional. These ideas are based on concentration-compactness arguments, where scaling techniques are relevant for proving that loss of mass at infinity does not occur. In our case we are able to prove an equivalent minimization and compactness result, although the scaling arguments with respect to the parameter $M$ are not appropriate at first sight in (3.6). We have

Theorem 4.1. Let us consider $M>0, J>0$ and $\mu \in[0,7 / 2)$. Let $\left\{\rho_{n}\right\}$ be a minimizing sequence for the problem (3.6) determined by $\mu, M$ and J. Then, there exists a sequence of shift vectors $\left\{a_{n}\right\} \in \mathbb{R}^{3}$ and a subsequence of $\left\{\rho_{n}\right\}$, again denoted by $\left\{\rho_{n}\right\}$, such that for any $\epsilon>0$ there exists $R>0$ such that

$$
\begin{aligned}
& \int_{a_{n}+B_{R}} \rho_{n}(x) \mathrm{d} x \geqslant M-\epsilon, \quad n \in \mathbb{N}, \\
& \rho_{n}\left(\cdot+a_{n}\right) \rightarrow \rho_{0} \quad \text { strongly in } \mathrm{L}^{1+\frac{2}{2 \mu+3}}\left(\mathbb{R}^{3}\right), \quad n \rightarrow \infty,
\end{aligned}
$$

and

$$
\int_{B_{R}} \rho_{0} \geqslant M-\epsilon .
$$


Finally,

$$
\nabla \phi_{T \rho_{n}} \rightarrow \nabla \phi_{\rho_{0}} \quad \text { strongly in } \mathrm{L}^{2}\left(\mathbb{R}^{3}\right), n \rightarrow \infty,
$$

and $\rho_{0}$ is a minimizer for (3.6). The set of minimizers is determined by $\left\{\rho_{0}(\cdot-y) ; y \in \mathbb{R}^{3}\right\}$, where $\rho_{0}$ is the unique spherically symmetric minimizer. Any minimizer verifies

$$
\rho_{0}(x)^{\frac{2}{2 \mu+3}}=\frac{3}{2 \mu+5} \frac{J^{\frac{2(\mu+1)}{2 \mu+3}}}{K^{\frac{3}{2 \mu+3}}}\left(-R_{M, J}^{\mu}\right)^{\frac{-2 \mu}{2 \mu+3}}\left(\frac{7-2 \mu}{3} \frac{R_{M, J}^{\mu}}{M}-\phi_{\rho_{0}}(x)\right)_{+}
$$

and

$$
\int_{\mathbb{R}^{3}} \rho_{0}(x)^{\frac{2 \mu+5}{2 \mu+3}} \mathrm{~d} x=\frac{\left(-R_{M, J}^{\mu}\right)^{\frac{3}{2 \mu+3}} J^{\frac{2 \mu+2}{2 \mu+3}}}{K^{\frac{3}{2 \mu+3}}} .
$$

The proof of Theorem 4.1 is a consequence of several intermediate results. The following lemma provides some properties of $R_{M, J}^{\mu}$. Let us remark that using Theorem 3.1 these properties are also satisfied by $I_{M, J}^{\mu}$.

Lemma 4.2. Let $\mu \in[0,7 / 2)$ and $M, J$ be positive constants. Then, the infimum values of (1.10) and (3.6) verify

(1) $I_{M, J}^{\mu}=R_{M, J}^{\mu}=M^{\frac{7}{3}-\frac{2 \mu}{3}} J^{\frac{2(\mu+1)}{3}} I_{1,1}^{\mu}$,

(2) $-\infty<I_{M, J}^{\mu}=R_{M, J}^{\mu}<0$.

Furthermore, if $f_{\mu}$ is a minimizer for $I_{1,1}^{\mu}$ then $I_{M, J}^{\mu}$ is achieved by $\breve{f}_{\mu}(x, v):=a f_{\mu}(b x, c v)$, where $a=J^{\mu+1} / M^{\mu}$, $b=J^{\frac{2(\mu+1)}{3}} / M^{\frac{2 \mu-1}{3}}$ and $c=M^{\frac{\mu-2}{3}} / J^{\frac{\mu+1}{3}}$. The relation between the minimizers is also satisfied for $R_{M, J}^{\mu}$.

Proof. The proof of (1) is based on the scaling $\breve{f}(x, v):=a f(b x, c v)$. If $a, b$ and $c$ are defined as in Lemma 4.2, then $E(\breve{f})=M^{\frac{7}{3}-\frac{2 \mu}{3}} J^{\frac{2(\mu+1)}{3}} E(f),\|\breve{f}\|_{L^{1}\left(\mathbb{R}^{6}\right)}=M\|f\|_{\mathrm{L}^{1}\left(\mathbb{R}^{6}\right)}$ and $\|\breve{f}\|_{\mathrm{L}^{1+1 / \mu}\left(\mathbb{R}^{6}\right)}=J\|f\|_{\mathrm{L}^{1+1 / \mu}\left(\mathbb{R}^{6}\right)}$. (1) is deduced from simple arguments.

To prove (2), let $\rho \in \mathrm{L}^{1}\left(\mathbb{R}^{3}\right) \cap \mathrm{L}^{\frac{2 \mu+5}{2 \mu+3}}\left(\mathbb{R}^{3}\right)$. By the Hölder and Hardy-Littlewood-Sobolev inequalities we have

$$
\|\rho\|_{L^{\frac{6}{5}}\left(\mathbb{R}^{3}\right)} \leqslant\|\rho\|_{L^{1}\left(\mathbb{R}^{3}\right)}^{\frac{7-2 \mu}{12}}\|\rho\|_{L^{\frac{2 \mu+5}{2 \mu+3}}\left(\mathbb{R}^{3}\right)}^{\frac{2 \mu+5}{12}}
$$

and

$$
\frac{1}{2} \int_{\mathbb{R}^{6}} \frac{\rho(x) \rho(y)}{|x-y|} \mathrm{d} x \mathrm{~d} y \leqslant C\|\rho\|_{\mathrm{L}^{\frac{6}{5}}\left(\mathbb{R}^{3}\right)}^{2} .
$$

Combining both estimates we find

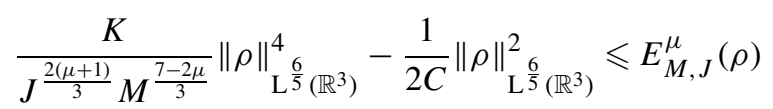

for any function $\rho \in \mathrm{L}_{+}^{1}\left(\mathbb{R}^{3}, M\right) \cap \mathrm{L}^{\frac{2 \mu+5}{2 \mu+3}}\left(\mathbb{R}^{3}\right)$ defined in problem (3.6). Thus, we obtain $R_{M, J}^{\mu}>-\infty$ because the left-hand side of (4.8) can be seen as a second degree polynomial in the variable $\|\rho\|_{L^{\frac{6}{5}}\left(\mathbb{R}^{3}\right)}^{2}$. To conclude, we have to prove $R_{M, J}^{\mu}<0$. Consider the scaled function $\breve{\rho}(x)=b^{3} \rho(b x)$, where $b$ is a positive constant. Clearly, $\breve{\rho}$ verifies $\|\breve{\rho}\|_{\mathrm{L}^{1}\left(\mathbb{R}^{3}\right)}=\|\rho\|_{\mathrm{L}^{1}\left(\mathbb{R}^{3}\right)}=M$ and

$$
E_{J}^{\mu}(\breve{\rho})=b^{2} K \frac{\left(\int_{\mathbb{R}^{3}} \rho(x)^{\frac{2 \mu+5}{2 \mu+3}} \mathrm{~d} x\right)^{\frac{2 \mu+3}{3}}}{J^{\frac{2(\mu+1)}{3}}}-\frac{1}{2} b \int_{\mathbb{R}^{6}} \frac{\rho(x) \rho(y)}{|x-y|} \mathrm{d} x \mathrm{~d} y .
$$


By choosing $b$ small enough we find a scaled function such that $E_{J}^{\mu}(\breve{\rho})<0$, which implies $R_{M, J}^{\mu}<0$. This ends the proof.

The boundedness of any minimizing sequence will be relevant for the minimization argument. This is what we state in the following result.

Corollary 4.3. Any minimizing sequence for (3.6) is uniformly bounded in $\mathrm{L}^{\frac{2 \mu+5}{2 \mu+3}}\left(\mathbb{R}^{3}\right)$.

Proof. Let $\left\{\rho_{n}\right\}$ be a minimizing sequence. (4.8) implies that any minimizing sequence is uniformly bounded in $\mathrm{L}^{\frac{6}{5}}\left(\mathbb{R}^{3}\right)$. Then, by using $(4.7)$ we also deduce that

$$
\frac{1}{2} \int_{\mathbb{R}^{6}} \frac{\rho_{n}(x) \rho_{n}(y)}{|x-y|} \mathrm{d} x \mathrm{~d} y
$$

is uniformly bounded. Finally, the definition of $E_{J}^{\mu}$ allows to conclude the proof.

The proof of Theorem 4.1 is also based on the fact that the minimizing sequence cannot vanish, as well as on the well-known compactness properties of the solution of the Poisson equation. Our next result shows an estimate which will confirm that the minimizing sequence does not vanish. Also, Lemma 4.4 concerns the compactness properties of the solution of the Poisson equation. Although, these results were already proved in [19], we write them here again for self-consistency (see [19] for more details). From now on, $B(a, R)$ denotes the ball centered in $a$ with radius $R$.

Lemma 4.4 (Corollary 3.6 and Lemma 3.7, Ref. [19]). Let $\rho_{n}$ be a minimizing sequence for (3.6). Then, there exist $\delta_{0}, R_{0}, n_{0} \in \mathbb{N}$ and a sequence of shift vectors $a_{n} \in \mathbb{R}^{3}$ such that

$$
\int_{B\left(a_{n}, R\right)} \rho_{n}(x) \mathrm{d} x \geqslant \delta_{0}, \quad n>n_{0}, R>R_{0} .
$$

Since $\left\{\rho_{n}\right\}$ is a bounded sequence in $\mathrm{L}^{\frac{2 \mu+5}{2 \mu+3}}\left(\mathbb{R}^{3}\right)$ for a subsequence such that

$$
\rho_{n} \rightarrow \rho \quad \text { weakly in } \mathrm{L}^{\frac{2 \mu+5}{2 \mu+3}}\left(\mathbb{R}^{3}\right) .
$$

We have

(1) For any $R>0$,

$$
\nabla \phi_{\chi_{B(0, R)} \rho_{n}} \rightarrow \nabla \phi_{\chi_{B(0, R)} \rho} \text { strongly in } \mathrm{L}\left(\mathbb{R}^{3}\right) .
$$

Here, $\chi_{B(0, R)}$ denotes the characteristic function in the ball $B(0, R)$.

(2) If in addition $\left\{\rho_{n}\right\}$ is bounded in $\mathrm{L}^{1}\left(\mathbb{R}^{3}\right), \rho \in \mathrm{L}^{1}\left(\mathbb{R}^{3}\right)$, and for any $\epsilon>0$ there exist $R>0$ and $n_{0} \in \mathbb{N}$ such that

$$
\int_{|x|>R}\left|\rho_{n}(x)\right| \mathrm{d} x \leqslant \epsilon, \quad \forall n \geqslant n_{0},
$$

then

$$
\nabla \phi_{\rho_{n}} \rightarrow \nabla \phi_{\rho} \quad \text { strongly in } \mathrm{L}^{2}\left(\mathbb{R}^{3}\right) .
$$

Proof of Theorem 4.1. We first deal with the existence of a minimum. The main idea is to prove that any minimizing sequence $\left\{\rho_{n}\right\}$ for (3.6) has a subsequence (up to translations) which is under the hypotheses of (2) in Lemma 4.4.

The boundedness of $\rho_{n}$ is deduced from Corollary 4.3. Now, we prove that for all $\epsilon$ there exist $R$ and $a_{n} \in \mathbb{R}^{3}$, $\forall n \in \mathbb{N}$, such that

$$
\int_{\mathbb{R}^{3}-B\left(a_{n}, R\right)} \rho_{n}(x) \mathrm{d} x \leqslant \epsilon .
$$


Let $\rho \in \mathrm{L}_{+}^{1}\left(\mathbb{R}^{3}, M\right) \cap \mathrm{L}^{\frac{2 \mu+5}{2 \mu+3}}\left(\mathbb{R}^{3}\right)$. Define $\rho_{i}, i=1,2,3$, as

$$
\rho=\chi_{B\left(0, R_{1}\right)} \rho+\chi_{B\left(0, R_{2}\right)-B\left(0, R_{1}\right)} \rho+\chi_{\mathbb{R}^{3}-B\left(0, R_{2}\right)} \rho=\rho_{1}+\rho_{2}+\rho_{3},
$$

where $\chi_{\Omega}$ is the characteristic function of the set $\Omega$. Setting

$$
\alpha_{i}=\frac{\int_{\mathbb{R}^{3}} \rho_{i}(x)^{\frac{2 \mu+5}{2 \mu+3}} \mathrm{~d} x}{\int_{\mathbb{R}^{3}} \rho(x)^{\frac{2 \mu+5}{2 \mu+3}} \mathrm{~d} x}, \quad \beta_{i}=\frac{\int_{\mathbb{R}^{3}} \rho_{i}(x) \mathrm{d} x}{\int_{\mathbb{R}^{3}} \rho(x) \mathrm{d} x} \quad \text { and } \quad F_{i, j}=\int_{\mathbb{R}^{6}} \frac{\rho_{i}(x) \rho_{j}(y)}{|x-y|} \mathrm{d} x \mathrm{~d} y
$$

for $i, j=1,2,3$, we observe that (4.10) is equivalent to proving that $\beta_{3}$ is small enough for an appropriate $R_{2}$. We have

$$
\begin{aligned}
E_{J}^{\mu}(\rho) & =\sum_{i=1}^{3} K \frac{\left(\int_{\mathbb{R}^{3}} \rho_{i}(x)^{\frac{2 \mu+5}{2 \mu+3}} \mathrm{~d} x\right)^{\frac{2 \mu+3}{3}}}{\left(\alpha_{i}^{\frac{\mu}{\mu+1}} J\right)^{\frac{2(\mu+1)}{3}}}+2 F_{i, i}-F_{1,2}-F_{2,3}-F_{1,3} \\
& =\sum_{i=1}^{3} E_{\alpha_{i}^{\mu}}^{\mu}\left(\rho_{i}\right)-F_{1,2}-F_{2,3}-F_{1,3} \\
& \geqslant \sum_{i=1}^{3} R_{\beta_{i} M, \alpha_{i}^{\mu+1} J}^{\frac{\mu}{\mu+1}}-F_{1,2}-F_{2,3}-F_{1,3} .
\end{aligned}
$$

Now, from Lemma 4.2

$$
\begin{aligned}
E_{J}^{\mu}(\rho) & \geqslant\left(\sum_{i=1}^{3}\left(\beta_{i} M\right)^{\frac{7}{3}-\frac{2 \mu}{3}}\left(\alpha_{i}^{\frac{\mu}{\mu+1}} J\right)^{\frac{2(\mu+1)}{3}} I_{1,1}^{\mu}\right)-F_{1,2}-F_{2,3}-F_{1,3} \\
& \geqslant M^{\frac{7}{3}-\frac{2 \mu}{3}} J^{\frac{2(\mu+1)}{3}} I_{1,1}^{\mu}\left(\sum_{i=1}^{3}\left(\beta_{i}^{\frac{7}{3}}\right)^{1-\frac{2 \mu}{7}} \alpha_{i}^{\frac{2 \mu}{3}}\right)-F_{1,2}-F_{2,3}-F_{1,3} .
\end{aligned}
$$

By Jensen's inequality we have

$$
\begin{aligned}
E_{J}^{\mu}(\rho) & \geqslant R_{M, J}^{\mu}\left(\sum_{i=1}^{3} \alpha_{i}\right)^{\frac{2 \mu}{3}}\left(\sum_{i=1}^{3} \beta_{i}^{\frac{7}{3}}\right)^{1-\frac{2 \mu}{7}}-F_{1,2}-F_{2,3}-F_{1,3} \\
& \geqslant R_{M, J}^{\mu}\left(\sum_{i=1}^{3} \beta_{i}^{\frac{7}{3}}\right)^{1-\frac{2 \mu}{7}}-F_{1,2}-F_{2,3}-F_{1,3}
\end{aligned}
$$

where we have only used that $\sum_{i=1}^{3} \alpha_{i}=1$. By using the estimate

$$
\sum_{i=1}^{3} \beta_{i}^{\frac{7}{3}} \leqslant 1-\frac{7}{3}\left(\beta_{1}+\beta_{2}\right) \beta_{3}
$$

we get,

$$
E_{J}^{\mu}(\rho) \geqslant R_{M, J}^{\mu}\left(1-\frac{7}{3}\left(\beta_{1}+\beta_{2}\right) \beta_{3}\right)^{1-\frac{2 \mu}{7}}-F_{1,2}-F_{2,3}-F_{1,3} .
$$

(4.11) and Lemma 4.2 now yield

$$
R_{(M, J)}^{\mu}-E_{J}^{\mu}(\rho) \leqslant R_{M, J}^{\mu}\left(1-\left(1-\frac{7}{3}\left(\beta_{1}+\beta_{2}\right) \beta_{3}\right)^{1-\frac{2 \mu}{7}}\right)+F_{1,2}+F_{2,3}+F_{1,3} .
$$

The first term in the right-hand side of this expression is estimated by using the inequality

$$
b^{\alpha}-a^{\alpha} \geqslant \alpha b^{\alpha-1}(b-a),
$$


valid for any $a, b>0$ and $0<\alpha<1$ (Theorem 41, Ref. [15]). In the same way, as proposed in [19], for $R_{2}>2 R_{1}$ we can estimate $F_{1,3}$ as follows

$$
F_{1,3} \leqslant C / R_{2} \text {. }
$$

We also have

$$
F_{1,2}+F_{2,3} \leqslant C\|\rho\|_{L^{\frac{2 \mu+5}{2 \mu+3}}\left(\mathbb{R}^{3}\right)}^{\frac{2 \mu+5}{6}}\left\|\nabla \phi_{\rho_{2}}\right\|_{\mathrm{L}^{2}\left(\mathbb{R}^{3}\right)} \leqslant C\left\|\nabla \phi_{\rho_{2}}\right\|_{\mathrm{L}^{2}\left(\mathbb{R}^{3}\right)} .
$$

Thus, $\rho$ verifies

$$
R_{M, J}^{\mu}-E_{J}^{\mu}(\rho) \leqslant R_{M, J}^{\mu}\left(\frac{7}{3}-\frac{2 \mu}{3}\right)\left(\beta_{1}+\beta_{2}\right) \beta_{3}+\frac{C}{R_{2}}+C\left\|\nabla \phi_{\rho_{2}}\right\|_{\mathrm{L}^{2}\left(\mathbb{R}^{3}\right)} .
$$

(4.9) claims the existence of a sequence of shift vectors $a_{n} \in \mathbb{R}^{3}$ such that

$$
\int_{B(R)} \rho_{n}\left(x-a_{n}\right) \mathrm{d} x \geqslant \delta_{0}, \quad n>n_{0}, R>R_{0},
$$

for some $\delta_{0}, R_{0}, n_{0} \in \mathbb{N}$. The sequence $\bar{\rho}_{n}(\cdot)=\rho_{n}\left(\cdot-a_{n}\right)$ also minimizes (3.6) due to the translation invariance of $E_{J}^{\mu}$. From the boundedness of that sequence in $\mathrm{L}^{\frac{2 \mu+5}{2 \mu+3}}\left(\mathbb{R}^{3}\right)$, we get the existence of a weakly convergent subsequence

$$
\bar{\rho}_{n}(\cdot)=\rho_{n}\left(\cdot-a_{n}\right) \rightarrow \rho_{0} \quad \text { weakly in } \mathrm{L}^{\frac{2 \mu+5}{2 \mu+3}}\left(\mathbb{R}^{3}\right) .
$$

Let $\epsilon>0$. Now we apply (4.12) to any $\tilde{\rho}_{n}$ with $R_{1}>R_{0}$ and find

$$
-R_{M, J}^{\mu} \frac{7-2 \mu}{3} \frac{\delta_{0}}{M} \beta_{3} \leqslant \frac{C}{R_{2}}+C\left\|\nabla \phi_{\bar{\rho}_{n, 2}}\right\|_{\mathrm{L}^{2}\left(\mathbb{R}^{3}\right)}+E_{J}^{\mu}\left(\bar{\rho}_{n}\right)-R_{M, J}^{\mu},
$$

for all $n>n_{0}$. (4.13) and Lemma 4.4(1) allow to choose $R_{1}$ sufficiently large to obtain $C\left\|\nabla \phi_{\bar{\rho}_{n, 2}}\right\|_{\mathrm{L}^{2}\left(\mathbb{R}^{3}\right)} \leqslant \epsilon / 4$, $\forall n>n_{1}>n_{0}$. Now, we fix $R_{1}$ such that $R_{2}>2 R_{1}$ and $R_{2}>4 C / \epsilon$. Finally, from the minimizing character of $\bar{\rho}_{n}$, there exists $n_{2} \in \mathbb{N}, n_{2} \geqslant n_{1}$, such that $E_{J}^{\mu}\left(\bar{\rho}_{n}\right)-R_{(M, J)}^{\mu} \leqslant \epsilon / 4, \forall n>n_{2}$. Hence, we conclude that

$$
\frac{7-2 \mu}{3} \frac{\delta_{0}}{M} \beta_{3} \leqslant \epsilon \quad \forall n>n_{2},
$$

which ends the proof of (4.10). The boundedness of $\bar{\rho}_{n}$ in $\mathrm{L}^{1}\left(\mathbb{R}^{3}\right)$ and Lemma 4.4 (2) lead to

$$
\nabla \phi_{\bar{\rho}_{n}} \rightarrow \nabla \phi_{\rho_{0}} \quad \text { strongly in } \mathrm{L}^{2}\left(\mathbb{R}^{3}\right) .
$$

This convergence property allows to conclude that $\bar{\rho}_{n}$ converges strongly in $\mathrm{L}^{\frac{2 \mu+5}{2 \mu+3}}\left(\mathbb{R}^{3}\right)$ to $\rho_{0}$ and, at the same time, that this function is a minimizer. The weakly lower semicontinuous character of the $L^{p}$-norms joint with (4.13) and the previous convergence gives

$$
E_{J}^{\mu}\left(\rho_{0}\right) \leqslant \liminf E_{J}^{\mu}\left(\bar{\rho}_{n}\right) .
$$

Since $\bar{\rho}_{n}$ is also a minimizing sequence we can assure that $\rho_{0}$ is a minimizer for (3.6). As consequence of the definition of $E_{J}^{\mu},\left\|\bar{\rho}_{n}\right\|_{\mathrm{L}^{\frac{2 \mu+5}{2 \mu+3}}\left(\mathbb{R}^{3}\right)}$ decreases to $\left\|\rho_{0}\right\|_{\mathrm{L}^{\frac{2 \mu+5}{2 \mu+3}}\left(\mathbb{R}^{3}\right)}$ as $n \rightarrow \infty$, in particular limsup $\left\|\bar{\rho}_{n}\right\|_{\mathrm{L}^{\frac{2 \mu+5}{2 \mu+3}}\left(\mathbb{R}^{3}\right)} \leqslant\left\|\rho_{0}\right\|_{\mathrm{L}^{\frac{2 \mu+5}{2 \mu+3}}\left(\mathbb{R}^{3}\right)}$. This implies along with (4.13) and the uniform convexity of $\mathrm{L}^{\frac{2 \mu+5}{2 \mu+3}}\left(\mathbb{R}^{3}\right)$ the strong convergence of $\bar{\rho}_{n}$ to $\rho_{0}$ in $\mathrm{L}^{\frac{2 \mu+5}{2 \mu+3}}\left(\mathbb{R}^{3}\right)$.

Once we have the existence of a minimum we analyze the properties of the minimizers. By scaling arguments we deduce

$$
R_{M, J}^{\mu}=E_{J}^{\mu}\left(\rho_{0}\right)=-K \frac{\left(\int_{\mathbb{R}^{3}} \rho_{0}(x)^{\frac{2 \mu+5}{2 \mu+3}} \mathrm{~d} x\right)^{\frac{2 \mu+3}{3}}}{J^{\frac{2(\mu+1)}{3}}}=\int_{\mathbb{R}^{6}} \frac{\rho_{0}(x) \rho_{0}(y)}{|x-y|} \mathrm{d} x \mathrm{~d} y,
$$


which proves (4.6). Let $\breve{\rho_{0}}(x)=b^{3} \rho_{0}(b x)$, where $b$ is a positive constant. Then, $\breve{\rho_{0}} \in \mathrm{L}_{+}^{1}\left(\mathbb{R}^{3}, M\right)$ and

$$
E_{J}^{\mu}\left(\breve{\rho_{0}}\right)=b^{2} K \frac{\left(\int_{\mathbb{R}^{3}} \rho_{0}(x)^{\frac{2 \mu+5}{2 \mu+3}} \mathrm{~d} x\right)^{\frac{2 \mu+3}{3}}}{J^{\frac{2(\mu+1)}{3}}}-b \frac{1}{2} \int_{\mathbb{R}^{6}} \frac{\rho_{0}(x) \rho_{0}(y)}{|x-y|} \mathrm{d} x \mathrm{~d} y .
$$

Since $\rho_{0}$ is a minimizer, (4.15) holds.

The Euler-Lagrange equation for any minimizer $\rho_{0}$ is given by

$$
\frac{2 \mu+5}{3} \frac{K}{J^{\frac{2 \mu+2}{3}}}\left(\int_{\mathbb{R}^{3}} \rho_{0}(x)^{\frac{2 \mu+5}{2 \mu+3}} \mathrm{~d} x\right)^{\frac{2 \mu}{3}} \rho_{0}(x)^{\frac{2}{2 \mu+3}}+\phi_{\rho_{0}}+\lambda \chi=\beta,
$$

where $\lambda$ and $\beta$ are the Lagrange multipliers corresponding to the positivity and the mass constraints respectively, and where

$$
\chi(x)= \begin{cases}0 & \text { if } \rho_{0}(x)>0 \\ \geqslant 0 & \text { if } \rho_{0}(x)=0 .\end{cases}
$$

By multiplying (4.16) by $\rho_{0}$ and integrating we find

$$
\left(\int_{\mathbb{R}^{3}} \rho_{n}(x)^{\frac{2 \mu+5}{2 \mu+3}} \mathrm{~d} x\right)^{\frac{2 \mu+3}{3}}+\int_{\mathbb{R}^{6}} \frac{\rho_{0}(x) \rho_{0}(y)}{|x-y|} \mathrm{d} x \mathrm{~d} y=\beta M .
$$

Combining (4.17) and (4.15) we deduce that $\beta=\left(\frac{7-2 \mu}{3}\right) \frac{R_{M, J}^{\mu}}{M}<0$. (4.16) and the fact that $\lim _{|x| \rightarrow \infty} \phi_{\rho_{0}^{*}}=0$ imply that $\operatorname{Supp}\left(\rho_{0}\right)=\left\{x ; \beta-\phi_{\rho_{0}} \geqslant 0\right\}$. As consequence, we deduce (4.5).

Now we will determine the set of minimizers of (3.6). Riesz's Theorem (see Theorem 3.7 Ref. [17]) applies to guarantee that the symmetric rearrangement $\rho_{0}^{*}$ of any minimizer $\rho_{0}$ is also a minimizer. In addition, it ensures that any minimizer is a space translation of $\rho_{0}^{*}$. Now, we prove that there exists a unique spherically symmetric minimizer for (3.6). By Lemma 4.2, we can equivalently write (4.5) as

$$
\rho_{0}^{*}(r:=|x|)=a\left(b-\phi_{\rho_{0}^{*}}(r)\right)_{+}^{\mu+3 / 2},
$$

where

$$
a=\left(\frac{3}{2 \mu+5}\right)^{\frac{2 \mu+3}{2}} J^{\frac{(\mu+1)(2 \mu+3)}{3}} M^{\frac{2 \mu(7-2 \mu)}{3(2 \mu+3)}} K^{-1}\left(-I_{1,1}^{\mu}\right)^{\mu}
$$

and

$$
b=\left(\frac{7-2 \mu}{3}\right) J^{\frac{2(\mu+1)}{3}} M^{\frac{4-2 \mu}{3}} I_{1, J}^{\mu} .
$$

Note that $\phi_{\rho_{0}^{*}}$ is radial since $\rho_{0}^{*}$ is radial. Then, $\phi_{\rho_{0}^{*}}$ is a solution to the Poisson equation (1.3) in radial coordinates

$$
\frac{1}{r^{2}}\left(r^{2} \phi_{\rho_{0}^{*}}(r)^{\prime}\right)^{\prime}=a\left(b-\phi_{\rho_{0}^{*}}(r)\right)_{+}^{\mu+3 / 2} \text {. }
$$

Equivalently, by setting $y(r)=\left(b-\phi_{\rho_{0}^{*}}\left(a^{\frac{-1}{2}} r\right)\right)$ we obtain

$$
\left(r^{2} y(r)^{\prime}\right)^{\prime}=-r^{2}(y(r))_{+}^{\mu+3 / 2} .
$$

Thus, $y(r)$ is the positive part of the solution to the Emden-Folder equation. The existence and uniqueness of solutions of the initial value problem associated with this equation are well known. Actually, it is clear that

$$
y(r)=\beta^{\frac{2}{\mu+1 / 2}} \varphi(\beta r),
$$

where $\beta$ is defined by the initial condition $\lim _{r \rightarrow 0} y(r)=\alpha$. In our case, $\beta$ is determined by the mass constraint since

$$
M=4 \pi \int_{r} \rho_{0}^{*}(r) \mathrm{d} r=4 \pi \int_{r}\left(b-\phi_{\rho_{0}^{*}}(r)\right)_{+}^{\mu+3 / 2} \mathrm{~d} r=4 \pi a^{-1 / 2} \int_{r}(y(r))_{+}^{\mu+3 / 2} \mathrm{~d} r .
$$


In particular, uniqueness ensures that $\rho_{0}^{*}$ is unique. Therefore, the set of minimizers of (3.6) is the orbit of the unique spherical symmetric minimizer because $E_{J}^{\mu}$ is invariant by translations. This concludes the proof of Theorem 4.1.

\section{Minimizing argument: relative compactness}

In this section we propose a new minimizing argument for the problems set in (1.10) which allows to establish that any minimizing sequence is relatively compact in $\mathrm{L}^{1}\left(\mathbb{R}^{6}\right)$ up to spatial translations. This argument is based on the sequence of equivalent problems introduced in Theorems 3.1 and 4.1.

Proof of Theorem 2.1. For simplicity, along the proof we shall denote all the subsequences with the same name of the original sequence. Let us consider an arbitrary minimizing sequence $f_{n}$ for the problem (1.10). Then, Theorem 3.1 states that $\rho_{n}(\cdot)=\int_{\mathbb{R}^{3}} f_{n}(\cdot, v) \mathrm{d} v$ is a minimizing sequence for (3.6). The application of Theorem 4.1 to $\rho_{n}$ leads to the existence of a subsequence of $f_{n}$ and $a_{n} \in \mathbb{R}^{3}$ such that $\bar{f}_{n}(\cdot, \cdot)=f\left(\cdot-a_{n}, \cdot\right)$ and $\bar{\rho}_{n}(\cdot)=\rho\left(\cdot-a_{n}\right)$ verifying the thesis of this result. Let us denote by $\rho_{M, J}$ the limit of $\bar{\rho}_{n}$.

The sequence $\bar{f}_{n}$ is also a minimizing sequence for $(1.10)$ because the $\mathrm{L}^{p}$ norms and the total energy functional are invariant under space translations. Then, by using (4.4) we easily deduce that $E_{\mathrm{KIN}}\left(\bar{f}_{n}\right)$ is uniformly bounded in $n$. Now, we are in a position to claim that $\bar{f}_{n}$ satisfies the hypotheses of the Dunford-Pettis theorem:

(1) $\left\{\bar{f}_{n}\right\}_{n \in \mathbf{N}}$ is bounded in $\mathrm{L}^{1}\left(\mathbb{R}^{6}\right)$.

(2) Let $A \in \mathbb{R}^{6}$ be a measurable set. Then, there is no concentration in $A$ since

$$
\int_{A} \bar{f}_{n} \mathrm{~d} x \mathrm{~d} v \leqslant\left\|\bar{f}_{n}\right\|_{\mathrm{L}^{1+1 / \mu}\left(\mathbb{R}^{6}\right)}|A|^{\mu+1} .
$$

(3) As a consequence of (4.1) and of the fact that $E_{\mathrm{KIN}}\left(\bar{f}_{n}\right)$ is uniformly bounded in $n$, there is no vanishing:

$$
\int_{\{|(x, v)|>R\}} \bar{f}_{n} \mathrm{~d} x \mathrm{~d} v \leqslant \epsilon_{(R)} .
$$

Hence, we can assume that there exists a function $f_{M}$ such that

$$
\bar{f}_{n} \rightarrow f_{M, J} \quad \text { weakly in } \mathrm{L}^{1}\left(\mathbb{R}^{6}\right) \text {. }
$$

We can check that $\int_{\mathbb{R}^{3}} f_{M, J}(\cdot, v) \mathrm{d} v=\rho_{M, J}$ by the weak convergence of $\bar{f}_{n}$ to $f_{M, J},(4.1),(4.2)$ and (4.3). These estimates imply that $\int_{\mathbb{R}^{3}} f_{M, J}(\cdot, v) \mathrm{d} v=\rho_{M, J}$ in $\mathrm{L}^{1}\left(\mathbb{R}^{3}\right)$.

It can be also obtained the existence of a subsequence such that

$$
\begin{aligned}
& \bar{f}_{n} \rightarrow f_{M, J} \quad \text { weakly in } \mathrm{L}^{1+1 / \mu}\left(\mathbb{R}^{6}\right), \text { for } \mu \in(0,7 / 2), \\
& \bar{f}_{n} \stackrel{*}{\rightarrow} f_{M, J} \quad \text { weak-* in } \mathrm{L}^{\infty}\left(\mathbb{R}^{6}\right), \text { for } \mu=0 .
\end{aligned}
$$

To deduce (5.2) we use the relative compactness arguments in $\mathrm{L}^{p}$ and the fact that $\bar{f}_{n}$ is bounded in $\mathrm{L}^{1+1 / \mu}$. Then, we can extract subsequences of $\left\{f_{n}\right\}$ converging to some function $g$ such that $g=f_{M, J}$.

Once we have proved (5.2), we may claim that $f_{M, J}$ is a minimum of (1.10). To this aim, we distinguish two different cases depending on the value of $\mu$ :

1) $\mu \in(0,7 / 2)$ : Combining (4.3), (5.1) and (5.2) we get

$$
\left\|f_{M, J}\right\|_{\mathrm{L}^{1}\left(\mathbb{R}^{6}\right)}=M, \quad E_{\mathrm{KIN}}\left(f_{M, J}\right) \leqslant \liminf E_{\mathrm{KIN}}\left(\bar{f}_{n}\right)
$$

and

$$
\left\|f_{M, J}\right\|_{\mathrm{L}^{1+1 / \mu}\left(\mathbb{R}^{6}\right)} \leqslant \liminf \left\|\bar{f}_{n}\right\|_{\mathrm{L}^{1+1 / \mu}\left(\mathbb{R}^{6}\right)} .
$$

These estimates together with (4.4) allow to conclude that $f_{M, J}$ is a minimizer for the problem

$$
\inf \left\{E(f) ; f \in \mathrm{L}_{+}^{1}\left(\mathbb{R}^{6}, M\right),\|f\|_{\mathrm{L}^{1+1 / \mu}\left(\mathbb{R}^{6}\right)} \leqslant J\right\} .
$$


The scaling property stated in Lemma 4.2(1) allows to ensure that the minimum of this problem coincides with the minimum for (1.10), since any minimizer is in $\mathrm{L}_{+}^{1+1 / \mu}\left(\mathbb{R}^{3}, J\right)$. In particular we have that

$$
J=\lim _{n \rightarrow \infty}\|\bar{f}\|_{\mathrm{L}^{1+1 / \mu}\left(\mathbb{R}^{6}\right)}=\left\|f_{M, J}\right\|_{\mathrm{L}^{1+1 / \mu}\left(\mathbb{R}^{6}\right)} .
$$

By the uniform convexity of $\mathrm{L}^{1+1 / \mu}$ (note that $\mu \in(0,7 / 2)$ ) the above identity and (5.2) imply the strong convergence in $\mathrm{L}^{1+1 / \mu}$ of $\bar{f}_{n}$ to $f_{M, J}$. Consequently, a subsequence of $\bar{f}_{n}$ exists such that

$$
\bar{f}_{n}(x, v) \rightarrow f_{M, J}(x, v) \quad \text { a.e. in } \mathbb{R}^{6} .
$$

The weak and a.e. convergence established in (5.1) and (5.3) respectively, allow to conclude the strong convergence in $\mathrm{L}^{1}$ of a subsequence of $\bar{f}_{n}$ to $f_{M, J}$, via Egorov's theorem. This justifies the notation $f_{M, J}$ and ends this part of the proof.

2) $\mu=0$ : Similarly, (4.3), (5.1) and (5.2) imply

$$
\left\|f_{M, J}\right\|_{\mathrm{L}^{1}\left(\mathbb{R}^{6}\right)}=M, \quad\left\|f_{M, J}\right\|_{\mathrm{L}^{\infty}\left(\mathbb{R}^{6}\right)} \leqslant 1 \quad \text { and } \quad E_{\mathrm{KIN}}\left(f_{M, J}\right) \leqslant E_{\mathrm{KIN}}\left(\bar{f}_{n}\right) .
$$

These estimates together with (4.4) imply that $f_{M}$ is a minimizer for (1.7). Thus, by Theorem $3.1 f_{M, J}$ is defined by (3.1) with associated density function $\rho_{M, J}$. Also, it is well known that $f_{M, J}$ has compact support (see [8]). Since $f_{M, J} \equiv J$ on its support and $\left\|\bar{f}_{n}\right\|_{\mathrm{L}^{\infty}\left(\mathbb{R}^{6}\right)} \leqslant J$, we have $f_{M}-\bar{f}_{n} \geqslant 0$ on $\operatorname{Supp}\left(f_{M, J}\right)$ and consequently

$$
\begin{aligned}
\left\|f_{M, J}-\bar{f}_{n}\right\|_{\mathrm{L}^{1}\left(\mathbb{R}^{6}\right)} & =\int_{\operatorname{Supp}\left(f_{M, J}\right)} f_{M, J}-\bar{f}_{n} \mathrm{~d} x \mathrm{~d} v+\int_{\mathbb{R}^{6}-\operatorname{Supp}\left(f_{M, J}\right)} \bar{f}_{n} \mathrm{~d} x \mathrm{~d} v \\
& =2 M-2 \int_{\operatorname{Supp}\left(f_{M, J}\right)} \bar{f}_{n} \mathrm{~d} x \mathrm{~d} v .
\end{aligned}
$$

This estimate clearly goes to 0 as $n \rightarrow \infty$ by using (5.1). This concludes the proof of Theorem 2.1 .

Remark 5.1. In the case $\mu=0$ the relative compactness cannot be deduced in $\mathrm{L}^{\infty}\left(\mathbb{R}^{3}\right)$. Let us consider a particular minimizing sequence for the problem (1.7) defined by $f_{n}(x, v)=f_{M, J}\left(\frac{n}{n+1} x, \frac{n+1}{n} v\right), n \in \mathbb{N}$, where $f_{M}$ is a minimum of (1.7) given by Theorem 2.1. It can be easily proved that $f_{n} \rightarrow f_{M, J}$ in $\mathrm{L}^{1}\left(\mathbb{R}^{6}\right)$ as $n \rightarrow \infty$, while

$$
\left\|f_{n}(x-k, v)-f_{M, J}(x, v)\right\|_{\mathrm{L}^{\infty}\left(\mathbb{R}^{6}\right)}=1, \quad \forall n \in \mathbb{N}, \forall k \in \mathbb{R}^{3} .
$$

Remark 5.2. Combining (3.1), (3.2), Theorems 3.1, 4.1 and Lemma 4.2 we get that the polytrope $v_{\mu}$ in $\Gamma_{M, J}^{\mu}$ is given by

$$
\nu_{0}(x, v)= \begin{cases}J & \text { if } \frac{7}{3} M^{\frac{4}{3}} J^{\frac{2}{3}}-\phi_{\nu_{0}}(x) \geqslant \frac{|v|^{2}}{2}, \\ 0 & \text { elsewhere }\end{cases}
$$

for $\mu=0$ and

$$
\tilde{v}_{\mu}(x, v)=\left(\frac{3}{2(\mu+1)} \frac{J^{\frac{(\mu+1)(3-2 \mu)}{3 \mu}}}{M^{\frac{7-2 \mu}{3}}\left(-I_{1,1}^{\mu}\right)}\right)^{\mu}\left(\frac{7-2 \mu}{3} M^{\frac{4-2 \mu}{3}} J^{\frac{2(\mu+1)}{3}} I_{1,1}^{\mu}-\phi_{v_{\mu}}(x)-\frac{|v|^{2}}{2}\right)_{+}^{\mu}
$$

for $\mu \in(0,7 / 2)$. This clearly shows the relation between the parameters $c$ and $E_{0}$ appearing in the definition of the polytrope with $M$ and $J$.

\section{Acknowledgements}

The authors would like to thank very much Luis Vega, Gerhard Rein, Yan Guo and Gershon Wolansky for fruitful discussions. 


\section{Appendix A. Polytropes and variational approaches}

In this appendix we will show that the minimizing problem of the energy-Casimir functional under mass constraints:

$$
C_{M}^{\mu}:=\inf \left\{Q_{\mu, 0}(f) ; f \in \mathrm{L}_{+}^{1}\left(\mathbb{R}^{6}, M\right)\right\},
$$

and the minimization problem of the energy functional under mass-Casimir constraints

$$
E_{M}^{\mu}:=\inf \left\{E(f) ; f \in \Lambda_{M}^{\mu}\right\},
$$

can be equivalently reduced to certain problems defined by (1.10), where the parameters $M$ and $J$ are linked. To this aim, we basically use scaling arguments. These simple techniques also help to clarify some other points such as: (a) the technical constraint $\mu \leqslant 3 / 2$ arising in the Casimir minimization problem; (b) the choice of the appropriate functional space (defining the admissible perturbations) to show the stability of a polytropic solution; or (c) whether the minimum of (A.2) can be reached by two functions with different orbits.

Our next result allows to read the Casimir minimization problem (A.1) in an equivalent form.

Lemma A.1. Let $\mu \in(0,3 / 2)$ and $M>0$. Then,

$$
C_{M}^{\mu}=\left(\frac{\mu}{\mu+1}\right) J_{(\mu, M)}^{1+1 / \mu}+I_{\left.M, J_{(\mu, M)}\right)}^{\mu},
$$

where

$$
J_{(\mu, M)}=\left(\frac{-3}{2(\mu+1) M^{\frac{7}{3}-\frac{2 \mu}{3}} I_{1,1}^{\mu}}\right)^{\frac{3 \mu}{(2 \mu-3)(\mu+1)}} .
$$

Furthermore, the minimizers of $C_{M}^{\mu}$ and $I_{M, J_{(\mu, M)}}^{\mu}$ coincide.

Proof. The proof is based on the following identity

$$
C_{M}^{\mu}=\inf \left\{\frac{\mu}{\mu+1} J^{1+1 / \mu}+M^{\frac{7}{3}-\frac{2 \mu}{3}} J^{\frac{2(\mu+1)}{3}} I_{1,1}^{\mu} ; J \in \mathbb{R}^{+}\right\} .
$$

By standard computations, we can prove that the minimum of (A.4) is reached when $J=J_{(\mu, M)}$ and, as consequence,

$$
C_{M}^{\mu}=\frac{\mu}{\mu+1} J_{(\mu, M)}^{1+1 / \mu}+I_{M, J_{(\mu, M)}}^{\mu} .
$$

Then, we have reduced the problem (A.1) to a particular case of (1.10), so that we can ensure that the minimizers are the same for both problems.

Remark A.2. In the above sections we have proved that the minimizers for $I_{M, J}^{\mu}$ exist for $0 \leqslant \mu<7 / 2$. However, when we are dealing with the Casimir functional we are only able to find such minimizers for $\mu<3 / 2$, because (A.4) shows that $C_{M}^{\mu}$ is bounded from below if and only if this condition holds. This explains the original restrictions appearing in the literature [11,19].

In order to avoid the artificial restriction $\mu<3 / 2$, it was proposed in $[12,14]$ to find the polytropic solutions as minimizers for the energy functional under the mass-Casimir constraint (A.2). In our next result we prove that (A.2) is also related to (1.10).

Lemma A.3. Let $\mu \in(0,7 / 2)$ and $M>0$. Then

$$
E_{M}^{\mu}=I_{a_{M}, b_{M}}^{\mu},
$$

where $a_{M}=2 M / 7$ and $b_{M}=(2 M(\mu+1) / 7)^{\frac{\mu}{\mu+1}}$. Furthermore, the minimizers for both problems coincide. 
Proof. Trivially, $E_{M}^{\mu} \leqslant I_{a_{M}, b_{M}}$, since $\Gamma_{a_{M}, b_{M}} \subset \Lambda_{M}^{\mu}$. In order to prove the reverse inequality we are going to show that any minimizer for $E_{M}^{\mu}$ (whose existence was proved in [12]) is in $\Gamma_{a_{M}, b_{M}}^{\mu}$. Let $f_{M}$ be such a minimizer. The scaled functions $\breve{f_{M}}(x, v):=a f_{M}(b x, c v) \in \Lambda_{M}^{\mu}$ lead to

$$
c=\frac{a^{1 / 3}}{M^{1 / 3} b}\left(a^{1 / \mu} \frac{\mu}{\mu+1}\left\|f_{M}\right\|_{\mathrm{L}^{1+1 / \mu}\left(\mathbb{R}^{6}\right)}^{1+1 / \mu}+\left(\frac{7}{2}-\mu\right)\left\|f_{M}\right\|_{\mathrm{L}^{1}\left(\mathbb{R}^{6}\right)}\right)^{1 / 3},
$$

where $a$ and $b$ are positive constants. The total energy associated with the scaled function depends on the parameters $a$ and $b$. Indeed,

$$
\begin{aligned}
E\left(\breve{f_{M}}\right)= & \frac{M^{5 / 3} b^{2}}{a^{2 / 3}\left(a^{1 / \mu} \frac{\mu}{\mu+1}\left\|f_{M}\right\|_{\mathrm{L}^{1+1 / \mu}\left(\mathbb{R}^{6}\right)}^{1+1 / \mu}+(7 / 2-\mu)\left\|f_{M}\right\|_{\mathrm{L}^{1}\left(\mathbb{R}^{6}\right)}\right)^{5 / 3}} E_{\mathrm{KIN}}\left(f_{M}\right) \\
& -\frac{M^{2} b}{\left(a^{1 / \mu} \frac{\mu}{\mu+1}\left\|f_{M}\right\|_{\mathrm{L}^{1+1 / \mu}\left(\mathbb{R}^{6}\right)}^{1+1 / \mu}+(7 / 2-\mu)\left\|f_{M}\right\|_{\mathrm{L}^{1}\left(\mathbb{R}^{6}\right)}\right)^{2}} E_{\mathrm{POT}}\left(f_{M}\right) .
\end{aligned}
$$

Considering the function $h: \mathbb{R}^{+} \times \mathbb{R}^{+} \rightarrow \mathbb{R}$ defined by $h(a, b):=E\left(\breve{f_{M}}\right)$ and the minimizing character of $f_{M}$ we have that $h$ admits a relative minimum in $(a, b)=(1,1)$. Then, we find

$$
\frac{\partial h}{\partial a}(1,1)=0, \quad \frac{\partial h}{\partial b}(1,1)=0 .
$$

By computing both equations we obtain

$$
\frac{1}{\mu+1}\left\|f_{M}\right\|_{\mathrm{L}^{1+1 / \mu}\left(\mathbb{R}^{6}\right)}^{1+1 / \mu}=\frac{2 M}{7} \quad \text { and } \quad E_{\mathrm{POT}}\left(f_{M}\right)=2 E_{\mathrm{KIN}}\left(f_{M}\right),
$$

which concludes the proof.

Remark A.4. Notice that (A.2) has a unique minimizer (up to translations). This completes the results established in [14].

Remark A.5. We also observe that Casimir minimization problems as well as energy minimization problems in massCasimir restricted spaces allow to study a particular subset of polytropes for which $c$ and $E_{0}-\alpha$ are connected.

The above results and the ideas developed in [8] to treat with the stability of the polytropic solution in the case $\mu=0$ have motivated the analysis of (1.10). From previous results in $[8,11,12]$, we can ensure the existence of minimizers for (1.10). However, we developed in this paper a constructive argument to solve these type of problems which is based on the equivalence between (1.10) and a problem for the corresponding densities.

\section{References}

[1] V.A. Antonov, Remarks on the problems of stability in stellar dynamics, Soviet Astron. AJ. 4 (1961) 859-867.

[2] V.A. Antonov, Solution of the problem of stability of a stellar system with the Emden density law and spherical velocity distribution, J. Leningrad Univ. Ser. Mekh. Astro. 7 (1962) 135-146.

[3] J. Barnes, J. Goodman, P. Hut, Dynamical instabilities in spherical stellar systems, Astrophys. J. 300 (1986) $112-131$.

[4] J. Batt, A survey of recent results in the investigation of the Vlasov-Poisson system and questions open for further research, in: L.L. Bonilla, B. Perthame, J. Soler, J.L. Vázquez (Eds.), European Workshop on Kinetic Equations, Book of Abstracts, Granada, Spain, 1996.

[5] J. Batt, W. Faltenbacher, E. Horst, Stationary spherically symmetric models in stellar dynamics, Arch. Rational Mech. Anal. 93 (2) (1986) 159-183.

[6] T. Cazenave, An Introduction to Nonlinear Schrödinger Equations, third ed., Textos de Métodos Matemáticos, vol. 26, Universidade Federal do Rio de Janeiro, Rio de Janeiro, RJ, 1996.

[7] T. Cazenave, P.L. Lions, Orbital stability of standing waves for some nonlinear Schrödinger equations, Commun. Math. Phys. 85 (1982) 549-561.

[8] J. Dolbeault, O. Sánchez, J. Soler, Asymptotic behaviour for the Vlasov-Poisson system in the stellar dynamics case, Arch. Rational Mech. Anal. 171 (2004) 301-327.

[9] M. Grillakis, J. Shatah, W. Strauss, Stability theory of solitary waves in the presence of symmetry, I, J. Func. Anal. 74 (1987) $160-197$.

[10] M. Grillakis, J. Shatah, W. Strauss, Stability theory of solitary waves in the presence of symmetry, II, J. Func. Anal. 94 (1990) 308-348. 
[11] Y. Guo, Variational method for stable polytropic galaxies, Arch. Rational Mech. Anal. 150 (1999) 209-224.

[12] Y. Guo, On the generalized Antonov's stability criterion, Contemp. Math. 150 (2000) 85-107.

[13] Y. Guo, G. Rein, Stable steady states in stellar dynamics, Arch. Rational Mech. Anal. 147 (1999) 225-243.

[14] Y. Guo, G. Rein, Isotropic steady states in galactic dynamics, Commun. Math. Phys. 209 (2001) 607-629.

[15] G. Hardy, J.E. Littlewood, G. Pólya, Inequalities, second ed., Cambridge Mathematical Press, Cambridge, 1952.

[16] M. Hénon, Numerical experiments on the stability of spherical stellar systems, Astronom. Astrophys. 24 (1973) $229-238$.

[17] E. Lieb, M. Loss, Analysis, second ed., American Mathematical Society, Providence, RI, 2001.

[18] P.L. Lions, B. Perthame, Propagation of moments and regularity for the 3-dimensional Vlasov-Poisson system, Invent. Math. 105 (2) (1991) 415-430.

[19] G. Rein, Reduction and a concentration-compactness principle for energy-Casimir functionals, SIAM J. Math. Anal. 33 (2001) 896-912.

[20] G. Rein, Stability of spherically symmetric steady states in galactic dynamics against general perturbations, Arch. Rational Mech. Anal. 161 (2002) 27-42.

[21] J. Schaeffer, Steady states in galactic dynamics, Arch. Rational Mech. Anal. 172 (2004) 1-19.

[22] Y.H. Wan, On nonlinear stability of isotropic models in stellar dynamics, Arch. Rational Mech. Anal. 147 (1999) $245-268$.

[23] G. Wolansky, On nonlinear stability of polytropic galaxies, Ann. Inst. H. Poincaré Anal. Non Linéaire 16 (1999) 15-48. 